\title{
On the Uniqueness Problem for Quadrature Domains
}

\author{
Yacin Ameur ${ }^{1} \cdot$ Martin Helmer ${ }^{2}$ (D) Felix Tellander $^{3}([)$ \\ Received: 18 June 2020 / Revised: 15 September 2020 / Accepted: 29 September 2020 / \\ Published online: 4 May 2021 \\ (C) The Author(s) 2021
}

\begin{abstract}
We study questions of existence and uniqueness of quadrature domains using computational tools from real algebraic geometry. These problems are transformed into questions about the number of solutions to an associated real semi-algebraic system, which is analyzed using the method of real comprehensive triangular decomposition.
\end{abstract}

Keywords Quadrature domain · Conformal mapping · Real comprehensive triangular decomposition

Mathematics Subject Classification 30C20 -31A25 $\cdot 14 \mathrm{P} 10 \cdot 68 \mathrm{~W} 30$

\section{Introduction}

This note is the result of investigations into an open uniqueness question for quadrature domains in the complex plane $\mathbf{C}$, which appears in papers such as $[21,24,44]$. After describing the problem and reviewing some known results, we will suggest and explore an approach based on methods from real algebraic geometry and symbolic computation.

To get started, it is convenient to fix some notation that will be used throughout.

Communicated by Darren Crowdy.

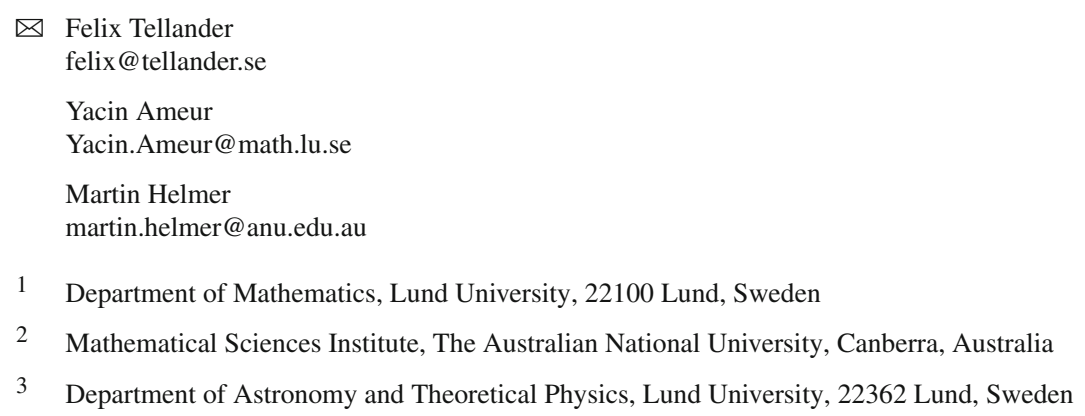


General notation. By a "domain" $\Omega$ we mean an open and connected subset of $\mathbf{C}$; we write $\bar{\Omega}$ for its closure, $\partial \Omega$ for its boundary, and $\Omega^{e}=\mathbf{C} \backslash \bar{\Omega}$ for its exterior. A bounded domain $\Omega$ is said to be "solid" if $\Omega^{e}$ is connected and $\partial \Omega=\partial\left(\Omega^{e}\right)$. Note that what we call solid domains are also referred to as "Carathéodory domains" in some sources.

We write " $d A$ " for the normalized Lebesgue measure in the plane $d A(z)=\frac{1}{\pi} d x d y$ (so the unit disc has area 1).

We denote by $L^{1}(\Omega)$ the usual $L^{1}$-space of functions on $\Omega$ that are integrable with respect to $d A$, and we write $A L^{1}(\Omega), H L^{1}(\Omega)$, and $S L^{1}(\Omega)$ for the subsets of $L^{1}(\Omega)$ consisting of all analytic, harmonic, and subharmonic functions, respectively.

Standard sets: $D(a, r)=\{z \in \mathbf{C} ;|z-a|<r\}, \mathbf{D}=D(0,1), \mathbf{T}=\partial \mathbf{D}$.

Differential operators: $\partial=\frac{1}{2}\left(\partial_{x}-i \partial_{y}\right), \bar{\partial}=\frac{1}{2}\left(\partial_{x}+i \partial_{y}\right), \Delta=4 \partial \bar{\partial}=\partial_{x}^{2}+\partial_{y}^{2}$.

Given an open set $\Omega \subset \mathbf{C}$, a subspace (or cone) $\mathcal{F} \subset L^{1}(\Omega)$, and a linear functional $\mu: \mathcal{F} \rightarrow \mathbf{C}$, we consider quadrature identities of the form

$$
\int_{\Omega} f d A=\mu(f), \quad f \in \mathcal{F} .
$$

The linear functional $\mu$ will always be a fixed measure or distribution of appropriate type with compact support in $\Omega$ (and defined on the appropriate test-class $\mathcal{F}$ ). Since we will frequently take $\mu$ to be a combination of point-evaluations, we stress that statements like $\mathcal{F} \subset L^{1}(\Omega)$ should not be taken literally, but rather in terms of the natural injective maps $\mathcal{F} \rightarrow L^{1}(\Omega)$.

If the above conditions hold, we say that $\Omega$ is a quadrature domain (or "q.d.") with data $(\mu, \mathcal{F})$, and we write $\Omega \in Q(\mu, \mathcal{F})$. We are mainly interested in the case $\mathcal{F}=A L^{1}(\Omega)$, but also $\mathcal{F}=H L^{1}(\Omega), \mathcal{F}=S L^{1}(\Omega)$ will play a role. In the last case, (1) must be replaced by the inequality

$$
\mu(f) \leq \int_{\Omega} f d A, \quad f \in S L^{1}(\Omega) .
$$

It is easy to see that $Q\left(\mu, S L^{1}\right) \subset Q\left(\mu, H L^{1}\right) \subset Q\left(\mu, A L^{1}\right)$ and that a solid domain belongs simultaneously to the classes $Q\left(\mu, H L^{1}\right)$ and $Q\left(\mu, A L^{1}\right)$.

The above classes are conveniently interpreted in terms of the logarithmic potentials

$$
U^{\mu}:=\ell * \mu, \quad U^{\Omega}:=U^{\mathbf{1}_{\Omega} d A}, \quad \text { where } \quad \ell(z):=\frac{1}{2} \log \frac{1}{|z|} .
$$

For example, we have that $\Omega \in Q\left(\mu, A L^{1}\right)$ if and only if $\partial U^{\mu}=\partial U^{\Omega}$ on $\Omega^{e}$ and $\Omega \in Q\left(\mu, S L^{1}\right)$ if and only if $U^{\mu}=U^{\Omega}$ on $\Omega^{e}$ and $U^{\mu} \geq U^{\Omega}$ on $\mathbf{C}$.

Given these proviso, we can formulate our basic problem in a succinct way (cf. [21]).

(Q). Determine whether or not there exists a functional $\mu$ such that the class $Q\left(\mu, A L^{1}\right)$ contains two distinct, solid domains.

Theorem 1.1 The following uniqueness results are known. 
(i) If $\Omega_{1}, \Omega_{2} \in Q\left(\mu, A L^{1}\right)$ are star-shaped with respect to a common point, then $\Omega_{1}=\Omega_{2}$.

(ii) If there exists a solid domain $\Omega \in Q\left(\mu, S L^{1}\right)$, then this $\Omega$ is the unique solid quadrature domain, even within the class $Q\left(\mu, A L^{1}\right)$.

(iii) If $\mu$ is a positive measure of total mass $m$ and if supp $\mu$ is contained in a disc of radius $r$ where $r^{2}<m$, then each solid domain $\Omega$ of class $Q\left(\mu, A L^{1}\right)$ is obtainable from $\mu$ by partial balayage, and so it belongs to $Q\left(\mu, S L^{1}\right)$.

Remark on the proof Part (i) is due to Novikov [33], cf. also [17,29]. Part (ii) was proved in Sakai's book [36], using the technique of partial balayage. An alternative proof is found in the paper [21] by Gustafsson. The statement (iii) was likewise proved by Sakai using partial balayage, see the papers $[27,35,38]$.

The "classical" setting corresponds to point-functionals, i.e., functionals $\mu$ of the form

$$
\mu(f)=\sum_{i=1}^{m} \sum_{j=0}^{n_{i}-1} c_{i j} f^{(j)}\left(a_{i}\right), \quad f \in A L^{1}(\Omega)
$$

where $a_{i}$ are some points in $\Omega$ and $c_{i j}$ some complex numbers. A quadrature domain of this type is said to be of order $n_{1}+\cdots+n_{m}$. When $\mu$ contains no derivatives, i.e., when $\mu(f)=\sum_{i=1}^{n} c_{i} f\left(a_{i}\right)$ we speak of a pure point-functional. (Cf. [15].)

Example 1.1 Theorem 1.1(ii) completely settles the uniqueness problem for subharmonic quadrature domains. The following example due to Gustafsson shows that the question (Q) for analytic test functions is of a different kind.

It is shown in [20, Sect. 4] that there exists a quadrature domain $\Omega$ having the appearance in Fig. 1, satisfying a three-point identity $\int_{\Omega} f d A=c_{1} f\left(a_{1}\right)+c_{2} f\left(a_{2}\right)+$ $c_{3} f\left(a_{3}\right)$ where $c_{1}, c_{2}, c_{3}>0$.

It is known (see e.g. [27, Thm. 2.1]) that if $\Omega \in Q\left(\mu, S L^{1}\right)$ and if $\partial \Omega$ has cusps, then those cusps must be contained in the convex hull of the nodes $a_{i}$. Hence the quadrature domain in Fig. 1 is not subharmonic.

Now fix a solid quadrature domain $\Omega \in Q\left(\mu, A L^{1}\right)$ containing the origin 0 . Let $\varphi: \mathbf{D} \rightarrow \Omega$ be the conformal map normalized by $\varphi(0)=0$ and $\varphi^{\prime}(0)>0$. Recall that $\Omega$ is uniquely determined by $\varphi$ via Riemann's mapping theorem.

Fig. 1 Gustafsson's example of a domain in

$Q\left(\mu, H L^{1}\right) \backslash Q\left(\mu, S L^{1}\right)$. The triangle depicts the convex hull of the nodes $a_{1}, a_{2}, a_{3}$

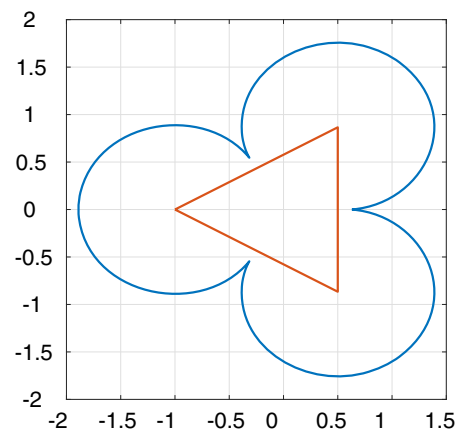


The following theorem, which gives a non-trivial relation for $\varphi$, will be the main tool in our subsequent investigations.

Theorem 1.2 Suppose that $\mu$ has compact support in $\Omega$ and let $v$ be the pullback of $\mu$ to $\mathbf{D}$, i.e., $v(g)=\mu\left(g \circ \varphi^{-1}\right)$ for $g \in A L^{1}(\mathbf{D})$. Then $\varphi$ satisfies the relation

$$
\varphi(z)=v_{\lambda}^{*}\left(\frac{z}{\overline{\varphi^{\prime}(\lambda)}(1-z \bar{\lambda})}\right), \quad z \in \mathbf{D}
$$

where $v_{\lambda}^{*}(g):=\overline{v_{\lambda}(\bar{g})}$ acts on integrable anti-analytic functions $g(\lambda)$.

Conversely, if $\varphi$ is any univalent solution to (2), then the domain $\Omega=\varphi(\mathbf{D})$ is of class $Q\left(\mu, A L^{1}\right)$.

This result is not very easy to spot in the literature, but it has in fact been noticed earlier in somewhat different guises. The first proof might be due to Davis, see [15, Ch. 14], cf. also [14, Sect. 5]. Since the result will be central for what follows, we include an alternative proof (that we have found independently) in Sect. 2.

In the special case of a pure point-functional $\mu(f)=\sum_{1}^{n} c_{i} f\left(a_{i}\right)$, the relation (2) takes the form

$$
\varphi(z)=\sum_{i=1}^{n} \frac{\bar{c}_{i}}{\bar{w}_{i}} \frac{z}{1-\bar{\lambda}_{i} z}, \quad w_{i}=\varphi^{\prime}\left(\lambda_{i}\right), \varphi\left(\lambda_{i}\right)=a_{i}
$$

which appears implicitly in e.g. the books [25,40,43].

The main idea behind our approach is to "solve" functional relations such as (3) by using techniques from algebraic geometry. To set up a suitable system of polynomial equations we differentiate (3) and substitute $z=\lambda_{j}$, giving

$$
w_{j}=\sum_{i=1}^{n} \frac{\bar{c}_{i}}{\bar{w}_{i}} \frac{1}{\left(1-\lambda_{j} \bar{\lambda}_{i}\right)^{2}}, \quad a_{j}=\sum_{i=1}^{n} \frac{\bar{c}_{i}}{\bar{w}_{i}} \frac{1}{1-\lambda_{j} \bar{\lambda}_{i}}, \quad j=1, \ldots, n
$$

where the unknown complex numbers $\lambda_{i}$ and $w_{i}$ are subject to the constraints

$$
\lambda_{1}=0, \quad\left|\lambda_{2}\right|<1, \quad \ldots, \quad\left|\lambda_{n}\right|<1, \quad w_{1}>0 .
$$

The appearance of inequalities and complex-conjugates means that we are considering the real semi-algebraic geometry of a particular system of rational functions. Such semi-algebraic systems, i.e. those having the special structure of (4), (5) have, to the best of our knowledge, not been systematically studied before.

It is of course possible that no univalent solution to (4), (5) exists; for example the quadrature identity $\int_{\Omega} f d A=f\left(a_{1}\right)+f\left(a_{2}\right)$ implies that $\Omega$ is the disjoint union $D\left(a_{1}, 1\right) \cup D\left(a_{2}, 1\right)$ if $\left|a_{1}-a_{2}\right| \geq 2$. However, after having studied exact solutions for many examples of lower order quadrature domains, we find "empirically" the pattern that the system tends to have at most one solution which may or may not give rise to a univalent mapping $\varphi$. The non-univalent solutions fail to be locally univalent, i.e., 
they (still, empirically) satisfy $\varphi^{\prime}=0$ somewhere in the disc D. For a different type of quadrature domains, not described by (4) and (5), a similar observation was made by Ullemar in [42].

Remark The non-univalent solutions $\varphi$ are believed to represent quadrature domains on Riemann surfaces with branch points. Such q.d.'s are studied in the references $[23,37,41]$.

Remark Note that our method relies on knowledge of all solutions to (4), (5). To find one or several approximate solutions, one can of course try to apply numerical methods, such as Newton's iterative method. By appropriately choosing different initial data, we may indeed obtain solutions by such methods in a relatively short time for $n$ up to 10 . However, since the number of solutions to the system is unknown, it is impossible to know when one has found all solutions, so this kind of information is of no use when studying the uniqueness question for quadrature domains. Another problem with a numerical approach is that systems such as (4), (5) tend to be quite sensitive to small perturbations of the quadrature data $\left\{c_{i}, a_{i}\right\}_{1}^{n}$.

Remark In connection with uniqueness problems in the gravi-equivalent sense, it is pertinent to point (besides the sources already mentioned) to the early works of Zidarov and Zhelev in the context of geophysics, see e.g. [45,46]. (We thank one of the referees for this remark.)

The literature on quadrature domains is vast, and we have at this stage omitted to mention several important aspects. A somewhat fuller picture is given in Sect. 7 , where we briefly compare a few of the more well-known techniques that have been developed over the years, such as Laplacian growth and Schottky-Klein functions.

To illustrate the challenges involved in studying the uniqueness of quadrature domains, we now give an example demonstrating the subtlety of the problem even for a q.d of order 2 .

Example 1.2 Let $\Omega_{1}$ be the solid quadrature domain obtained from a monopole with charge $1 / 2$ and a dipole with strength $\sqrt{3} / 18$ placed at the origin, i.e.

$$
\int_{\Omega_{1}} f d A=\frac{1}{2} f(0)+\frac{\sqrt{3}}{18} f^{\prime}(0), \quad f \in A L^{1}\left(\Omega_{1}\right) .
$$

For quadrature domains of this type, Aharonov and Shapiro have proved uniqueness in [2]; in fact $\Omega_{1}$ is determined as the image of $\mathbf{D}$ under the conformal map $p(z)=$ $\sqrt{3}\left(2 z+z^{2}\right) / 6$. The boundary $\partial \Omega_{1}$ is a cardioid with a cusp at $p(-1)=-1 / \sqrt{12}$, see Fig. 2.

Let us now construct a similar q.d. $\tilde{\Omega}_{2}$ but only using point charges,

$$
\int_{\tilde{\Omega}_{2}} f d A=-\frac{1}{2} f(0)+f\left(a_{2}\right), \quad f \in A L^{1}\left(\tilde{\Omega}_{2}\right) .
$$

Clearly both $\Omega_{1}$ and $\tilde{\Omega}_{2}$ have area $1 / 2$. We shall choose the parameter $a_{2}$ real, such that the boundary of $\tilde{\Omega}_{2}$ has a cusp. 


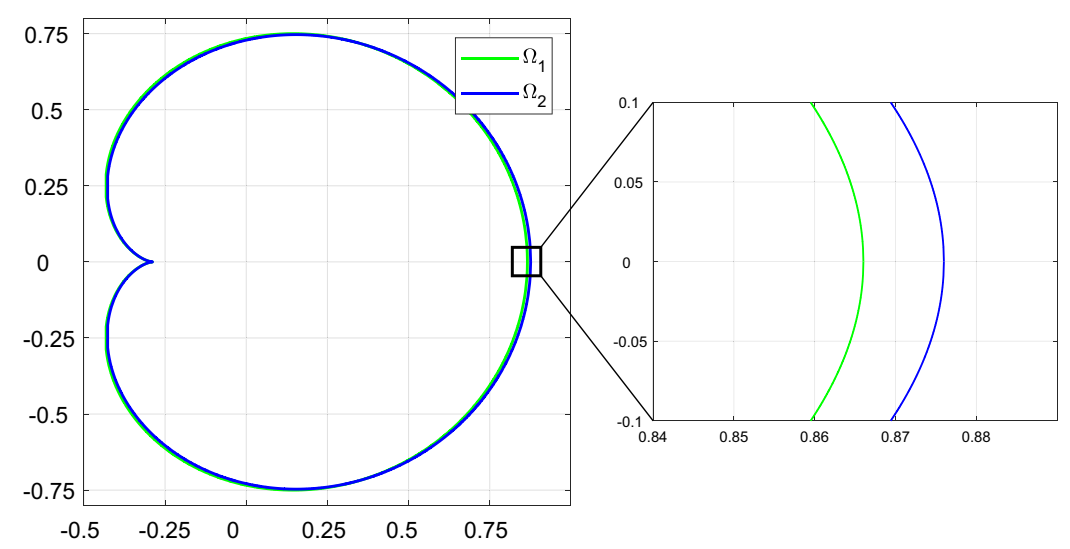

Fig. 2 The domains $\Omega_{1}$ and $\Omega_{2}$

Since the parameters $c_{i}, w_{i}, \lambda_{i}$ in (3) must be real in this case, the conformal map $\tilde{\varphi}: \mathbf{D} \rightarrow \tilde{\Omega}_{2}$ takes the form

$$
\tilde{\varphi}: z \mapsto \frac{c_{1} z}{w_{1}}+\frac{c_{2}}{w_{2}} \frac{z}{1-\lambda_{2} z}
$$

where $c_{1}=-1 / 2$ and $c_{2}=1, \lambda_{2}$ the pre-image of $a_{2}$, and $w_{1}=\tilde{\varphi}^{\prime}(0), w_{2}=\tilde{\varphi}^{\prime}\left(\lambda_{1}\right)$.

Writing $v=-9+18 i \sqrt{2}$, we find that there is a unique choice of $a_{2}$ producing a cusp, namely

$$
a_{2}=\frac{1}{2 v^{1 / 3}} \sqrt{-v^{1 / 3}\left(3 i \sqrt{3} v^{2 / 3}-27 i \sqrt{3}+3 v^{2 / 3}+4 v^{1 / 3}+27\right)} \approx 0.1316
$$

The remaining parameters in the mapping function may be obtained by solving the system corresponding to (6) using the method of "RCTD" described in Sect. 4. The result is

$$
\begin{aligned}
& \lambda_{2}=\frac{3 a_{2}}{\sqrt{3 a_{2}^{2}+3}} \approx 0.2261, \\
& w_{2}=\frac{-c_{2} \lambda_{2}{ }^{3}\left(-\lambda_{2}{ }^{2} c_{1}+\lambda_{2}{ }^{2} c_{2}+a_{2}{ }^{2}\right)}{a_{2}\left(-c_{1} \lambda_{2}{ }^{6}+c_{2} \lambda_{2}{ }^{6}+\lambda_{2}{ }^{4} a_{2}{ }^{2}+2 c_{1} \lambda_{2}{ }^{4}-2 \lambda_{2}{ }^{2} a_{2}{ }^{2}-\lambda_{2}{ }^{2} c_{1}-\lambda_{2}{ }^{2} c_{2}+a_{2}{ }^{2}\right)} \approx 0.6674, \\
& w_{1}=\frac{c_{1} \lambda_{2}\left(\lambda_{2}{ }^{2}-1\right) w_{2}}{w_{2} \lambda_{2}{ }^{2} a_{2}-w_{2} a_{2}+\lambda_{2} c_{2}} \approx 0.5016 .
\end{aligned}
$$

This gives a cusp at

$$
\tilde{\varphi}(-1)=-\frac{c_{1}}{w_{1}}-\frac{c_{2}}{w_{2}} \frac{1}{1+\lambda_{2}} \approx-0.2253
$$


A translate of $\tilde{\Omega}_{2}$ by $\alpha:=p(-1)-\tilde{\varphi}(-1) \approx-0.06333$ leads to a domain $\Omega_{2}=$ $\tilde{\Omega}_{2}+\alpha$ having a cusp at the point $p(-1)$ and satisfying the quadrature identity

$$
\int_{\Omega_{2}} f d A=-\frac{1}{2} f(\alpha)+f\left(a_{2}+\alpha\right), \quad f \in A L^{1}\left(\Omega_{2}\right) .
$$

The resemblance between $\Omega_{1}$ and $\Omega_{2}$ (Fig. 2) is striking, even though they admit completely different quadrature identities. The similarity between $\Omega_{1}$ and $\Omega_{2}$ indicates that their potentials should be similar, and in terms of numerical values they are. But there is one essential difference between the two: the potential of $\Omega_{1}$ is exactly determined by two terms in its multipole expansion while the potential for $\Omega_{2}$ needs the entire infinite series. In detail we have

$$
\begin{aligned}
& U^{\Omega_{1}}(z)=\frac{\frac{1}{2}}{2 \pi} \log \frac{1}{|z|}+\frac{\frac{\sqrt{3}}{18}}{2 \pi} \operatorname{Re}\left(\frac{1}{z}\right) \\
& U^{\Omega_{2}}(z) \approx \frac{\frac{1}{2}}{2 \pi} \log \frac{1}{|z|}+\frac{0.09998}{2 \pi} \operatorname{Re}\left(\frac{1}{z}\right)+\cdots
\end{aligned}
$$

and for comparison note $\sqrt{3} / 18 \approx 0.09623$. From this example we see that two very similar domains may have fundamentally different potentials.

\section{The Master Formula}

As previously stated, Theorem 1.2 appears (in equivalent form) in [15, Eq. (14.12)]. We shall here give a different derivation.

Consider the univalent map $\varphi: \mathbf{D} \rightarrow \Omega$ normalized by $\varphi(0)=0$ and $\varphi^{\prime}(0)>0$ where $\Omega \in Q\left(\mu, A L^{1}\right)$ and where the distribution $\mu$ is assumed to be of compact support in $\Omega$. Our point of departure is Poisson's equation

$$
\Delta U^{\mu}=-\mu .
$$

Taking the distributional $\partial$-derivative of $-4 U^{\mu}$ we obtain the Cauchy transform

$$
\mathcal{C} \mu(z):=\mu * k(z)=\mu\left(k_{z}\right),
$$

where $k(\lambda):=\lambda^{-1}$ denotes the Cauchy kernel and $k_{z}(\lambda):=k(z-\lambda)$. Since $-4 \partial U^{\mu}=$ $\mathcal{C} \mu$, (2) says that $\bar{\partial} \mathcal{C} \mu=\mu$. In particular $\mathcal{C} \mu$ is holomorphic on $\mathbb{C} \backslash \operatorname{supp} \mu$. Taking $f=k_{z}(z \notin \Omega)$ in the quadrature identity (1) we see that

$$
\partial U^{\Omega}=\partial U^{\mu} \quad \text { on } \quad \mathbf{C} \backslash \Omega .
$$

In fact, an application of Bers' approximation theorem from [4] shows that (7) is equivalent to (1). Now consider the "Schwarz potential" $u$ defined by

$$
u=\mathcal{C}\left(\mathbf{1}_{\Omega}-\mu\right)=-4 \partial\left(U^{\Omega}-U^{\mu}\right)
$$


which is zero for $z \in \Omega^{e}$. Using the continuity of $\partial U^{\Omega}$ we get $u=0$ also on $\partial \Omega$. Moreover, Poisson's equation gives $\bar{\partial} u=\mathbf{1}_{\Omega}$ on $\mathbf{C} \backslash$ supp $\mu$. Hence the function

$$
S(z):=\bar{z}-u(z)
$$

is holomorphic on $\Omega \backslash \operatorname{supp} \mu$ and continuous up to the boundary $\partial \Omega$, while satisfying $S(z)=\bar{z}$ for $z \in \partial \Omega$. This determines $S$ as the Schwarz function for the boundary curve $\partial \Omega$, cf. $[15,40]$.

The following lemma is well-known, see e.g. [15,40].

Lemma 2.1 The conformal mapping $\varphi: \mathbf{D} \rightarrow \Omega$ extends holomorphically across $\mathbf{T}$ to an analytic function on the disk $D(0, R)$ for some $R>1$.

Proof As we saw above, the function $S \circ \varphi$ is defined and holomorphic in some annulus $1-\epsilon<|z|<1$, continuous up to the boundary and satisfies $S(\varphi(z))=\overline{\varphi(z)}$ when $z \in \mathbf{T}$. Likewise, the function $\varphi^{*}(z)=\overline{\varphi(\bar{z})}$ is holomorphic in $\mathbf{D}$ and continuous up to the boundary, and we have the relation

$$
S(\varphi(z))=\varphi^{*}(1 / z), \quad z \in \mathbf{T} .
$$

Now, $\varphi^{*}(1 / z)$ is holomorphic in the exterior of $\mathbf{D}$, so the above formula shows that the functions $S \circ \varphi(z)$ and $\varphi^{*}(1 / z)$ are analytic continuations of each other across the circle $\mathbf{T}$. In particular, $\varphi^{*}(1 / z)$ is analytically continuable inwards across $\mathbf{T}$, which means that $\varphi^{*}$ as well as $\varphi$ are analytically continuable outwards across $\mathbf{T}$, to some $\operatorname{disc} D(0, R)$ with $R>1$.

Lemma 2.2 We have that $\mathcal{C}\left[\overline{\varphi^{\prime}} \cdot \mathbf{1}_{\mathbf{D}}\right](z)=\overline{\varphi(z)}, z \in \mathbf{T}$.

Remark For an absolutely continuous measure $v=f d A$, we prefer to denote its Cauchy transform by $\mathcal{C} f$ rather than $\mathcal{C} v$.

Proof of Lemma 2.2 Fix a point $z=e^{i \theta} \in \mathbf{T}$ and a positive number $r<1$ and put

$$
I_{r}=\mathcal{C}\left[\overline{\varphi^{\prime}} \cdot \mathbf{1}_{\mathbf{D}}\right](r z)=\int_{\mathbf{D}} \frac{\overline{\varphi^{\prime}(\lambda)}}{r e^{i \theta}-\lambda} d A(\lambda)=r e^{-i \theta} \int_{r \mathbf{D}} \frac{\overline{\varphi^{\prime}\left(r \zeta e^{i \theta}\right)}}{1-\zeta} d A(\zeta) .
$$

Set $\varphi(\lambda)=\sum_{j=0}^{\infty} c_{j} \lambda^{j}$, where $\sum_{j=0}^{\infty}\left|c_{j}\right|<\infty$ by Lemma 2.1. Inserting the expansion $1 /(1-\zeta)=\sum \zeta^{j}$ we find that

$$
I_{r}=\sum_{j=1}^{\infty} \bar{c}_{j}\left(r e^{-i \theta}\right)^{j} r^{2 j}
$$

Since $\sum\left|c_{j}\right|<\infty$ we may pass to the limit as $r \nearrow 1$, leading to

$$
\lim _{r \rightarrow 1} I_{r}=\lim _{r \rightarrow 1} \sum_{j=1}^{\infty} \bar{c}_{j}\left(r e^{-i \theta}\right)^{j} r^{2 j}=\sum_{j=1}^{\infty} \bar{c}_{j}\left(e^{-i \theta}\right)^{j}=\overline{\varphi(z)}
$$

The proof of the lemma is complete. 
Proof of Theorem 1.2 The quadrature identity (1) pulls back to

$$
\int_{\Omega} f d A=\int_{\mathbf{D}}(f \circ \varphi) \cdot\left|\varphi^{\prime}\right|^{2} d A=v(f \circ \varphi)
$$

where $\nu(g)=\mu\left(g \circ \varphi^{-1}\right)$.

Given an arbitrary $f \in A L^{1}(\Omega)$ we define a function $g \in A L^{1}(\mathbf{D})$ by

$$
g=(f \circ \varphi) \cdot \varphi^{\prime}
$$

The identity (8) can be written as

$$
\int_{\mathbf{D}} g \overline{\varphi^{\prime}} d A=v\left(\frac{g}{\varphi^{\prime}}\right)
$$

Now fix a point $z \in \mathbf{T}$ and choose $g$ to be the Cauchy-kernel $g=k_{z}$. With this choice, (9) takes the form

$$
\mathcal{C}\left[\overline{\varphi^{\prime}} \cdot \mathbf{1}_{\mathbf{D}}\right](z)=\mathcal{C}\left[\frac{1}{\varphi^{\prime}} \cdot v\right](z), \quad z \in \mathbf{T} .
$$

By Lemma 2.2 this is equivalent to

$$
\overline{\varphi(z)}=\mathcal{C}\left[\frac{1}{\varphi^{\prime}} \cdot v\right](z)=v_{\lambda}\left(\frac{1}{\varphi^{\prime}(\lambda)(1 / \bar{z}-\lambda)}\right), \quad z \in \mathbf{T} .
$$

Taking complex-conjugates and considering the analytic continuation to $\mathbf{D}$ we obtain

$$
\varphi(z)=v_{\lambda}^{*}\left[\frac{z}{\overline{\varphi^{\prime}(\lambda)}(1-z \bar{\lambda})}\right], \quad z \in \mathbf{D},
$$

as desired.

Conversely, if $\varphi$ is univalent (and normalized) in D and satisfies (10), we may read backwards and deduce (8), so $\Omega=\varphi(\mathbf{D})$ belongs to $Q\left(\mu, A L^{1}\right)$ where $\mu$ is the push-forward of $v$.

We conclude this section with three examples of applications of Theorem 1.2, which are known from the literature on quadrature domains.

Example 2.1 Let us compare Theorem 1.2 with the computations in Shapiro's book [40, Prop. 3.2]. For this purpose we fix a pure point functional $\mu=\sum_{i=1}^{n} c_{i} \delta_{a_{i}}$, which pulls back to

$$
v(g)=\mu\left(g \circ \varphi^{-1}\right)=\sum_{i=1}^{n} c_{i} g\left(\varphi^{-1}\left(a_{i}\right)\right)=\sum_{i=1}^{n} c_{i} g\left(\lambda_{i}\right), \quad \lambda_{i}=\varphi^{-1}\left(a_{i}\right)
$$


By Theorem 1.2 we know that an arbitrary solid domain $\Omega \in Q\left(\mu, A L^{1}\right)$ is of the form $\Omega=\varphi(\mathbf{D})$ where $\varphi$ is univalent and normalized and satisfies

$$
\varphi(z)=\sum_{i=1}^{n} \frac{\bar{c}_{i}}{\overline{\varphi^{\prime}\left(\lambda_{i}\right)}} \frac{z}{1-z \bar{\lambda}_{i}}
$$

Given such a $\varphi$, we put $S(\varphi(z)):=\varphi^{*}(1 / z)$ and note that $S$ is the Schwarz function for $\partial \Omega$. In view of (11), $S$ is a meromorphic function with simple poles at $z=a_{j}$. As $z \rightarrow \lambda_{j}$ the dominant term in $S \circ \varphi$ satisfies

$$
S(\varphi(z)) \sim \frac{c_{j}}{\varphi^{\prime}\left(\lambda_{j}\right)} \frac{1}{z-\lambda_{j}} .
$$

From this we get that (as $z \rightarrow a_{j}$ )

$$
S(z) \sim \frac{c_{j}}{\varphi^{\prime}\left(\lambda_{j}\right)} \frac{1}{\varphi^{-1}(z)-\lambda_{j}}=\frac{c_{j}}{\varphi^{\prime}\left(\lambda_{j}\right)} \frac{z-a_{j}}{\varphi^{-1}(z)-\varphi^{-1}\left(a_{j}\right)} \frac{1}{z-a_{j}} \sim \frac{c_{j}}{z-a_{j}} .
$$

The residues of $S$ are thus just $\operatorname{Res}\left(S ; a_{j}\right)=c_{j}$ for all $j$. Since $S(z)=\bar{z}$ on $\partial \Omega$, an application of Green's theorem and the Residue theorem now gives

$$
\int_{\Omega} f d A=\frac{1}{2 \pi i} \int_{\Gamma} f(z) \bar{z} d z=\frac{1}{2 \pi i} \int_{\Gamma} f(z) S(z) d z=\sum c_{j} f\left(a_{j}\right)
$$

where $\Gamma$ is the positively oriented boundary of $\Omega$. We have shown again that $\Omega \in$ $Q\left(\mu, A L^{1}\right)$.

We remark that a similar proof applied to a more general point functional $\mu(f)=$ $\sum c_{i j} f^{(j)}\left(a_{i}\right)$ gives the well known result (see [26, Thm. 3.3.1]) that a solid domain $\Omega$ is a quadrature domain of finite order if and only if each conformal map $\varphi: \mathbf{D} \rightarrow \Omega$ is a rational function, if and only if the Schwarz function of $\partial \Omega$ extends to a meromorphic function in $\Omega$.

Example 2.2 Let $\mu$ be a linear combination of a monopole and a dipole at the origin, i.e. $\mu(f)=M_{0} f(0)+M_{1} f^{\prime}(0)$. The action of the pullback is then given by

$$
v(g)=\mu\left(g \circ \varphi^{-1}\right)=M_{0} g(0)+\frac{M_{1}}{\varphi^{\prime}(0)} g^{\prime}(0), \quad g \in A L^{1}(\mathbf{D}) .
$$

Applying Theorem 1.2, we find that a normalized conformal map $\varphi: \mathbf{D} \rightarrow \Omega$, where $\Omega \in Q\left(\mu, A L^{1}\right)$, must satisfy

$$
\overline{\varphi(z)}=\left(\frac{1}{\varphi^{\prime}(0)} M_{0}-\frac{\varphi^{\prime \prime}(0)}{\varphi^{\prime}(0)^{3}} M_{1}\right) \bar{z}+\frac{M_{1}}{\varphi^{\prime}(0)^{2}} \bar{z}^{2} .
$$

Hence $\varphi(z)$ is a polynomial of degree two. To determine this polynomial, we need to determine the derivatives $\varphi^{\prime}(0), \varphi^{\prime \prime}(0)$. The computation is postponed to Subsect. 5.1, after we have discussed some algebraic prerequisites. 
Fig. 3 The q.d. $\Omega$ generated by the linear density $d \mu(x)=\mathbf{1}_{[-1,1]}(x) d x$

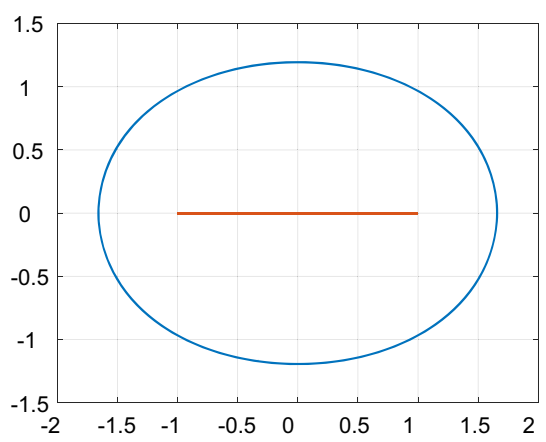

Example 2.3 Following Davis [15, pp. 162-166] we now take $\mu$ be a line charge with linear density $h$ on the segment $[-a, a]$ of the $x$-axis, i.e.,

$$
\mu(f)=\int_{-a}^{a} f(x) h(x) d x .
$$

Suppose that $\Omega=\varphi(\mathbf{D}) \in Q\left(\mu, A L^{1}\right)$; the pullback $v$ by $\varphi$ is then given by

$$
\begin{gathered}
v(g)=\mu\left(g \circ \varphi^{-1}\right)=\int_{-a}^{a} g\left(\varphi^{-1}(x)\right) h(x) d x=\int_{-\lambda}^{\lambda} g(w) h(\varphi(w)) \varphi^{\prime}(w) d w, \\
\lambda=\varphi^{-1}(a) .
\end{gathered}
$$

Applying Theorem 1.2 we see that $\varphi$ must satisfy the functional equation (cf. [15, Eq. (14.25)])

$$
\overline{\varphi(z)}=\bar{z} \int_{-\lambda}^{\lambda} \frac{h(\varphi(w))}{1-\bar{z} w} d w
$$

In particular, if we specialize to a uniform line charge $h \equiv 1$, we obtain

$$
\varphi(z)=\log \left(\frac{1+z \lambda}{1-z \lambda}\right) .
$$

This relation was found by Davis (see Eq. (14.13)), where it is also shown that if $a=1$ then $\lambda=\sqrt{\tanh (1 / 2)}=.6798 \cdots<1$. It is then easy to see that the above map $\varphi$ is well-defined by choosing the standard branch of the logarithm. We have shown that there exists a unique solid domain $\Omega$ in the class $Q\left(\mathbf{1}_{[-1,1]}(x) d x, A L^{1}\right)$; a picture is given in Fig. 3.

\section{Schur-Cohn's Test}

As stated earlier, the mapping problem (3) may have non-univalent solutions $\varphi$. The Schur-Cohn test provides a convenient way of discarding such $\varphi$ that fail to be locally 
univalent. One might hope that this procedure would leave us with at most one univalent $\varphi$, thus settling the uniqueness problem. As we will see later, this is indeed the case for a large class of examples.

The Schur-Cohn test is well known and quite elementary, see [28]. For reasons of completeness, we have found it convenient to briefly review the main ideas behind it here. In what follows, we let $\mathcal{P}_{n}$ denote the space of all polynomials $p$ of degree at $\operatorname{most} n$,

$$
p(z)=a_{0}+a_{1} z+\cdots+a_{n} z^{n}, \quad a_{0}, \ldots, a_{n} \in \mathbb{C} .
$$

Lemma 3.1 If $n \geq 1$ then

(i) if $\left|a_{0}\right|>\left|a_{1}\right|+\cdots+\left|a_{n}\right|$ then $p(z) \neq 0$ for all $z$ with $|z| \leq 1$,

(ii) if $p(z) \neq 0$ for all $z$ with $|z| \leq 1$ then $\left|a_{0}\right|>\left|a_{n}\right|$.

Proof (i) If $\left|a_{0}\right|>\left|a_{1}\right|+\cdots+\left|a_{n}\right|$ then $\left|a_{1} z+\cdots+a_{n} z^{n}\right| \leq\left|a_{1}\right|+\cdots+\left|a_{n}\right|<\left|a_{0}\right|$ for $|z| \leq 1$, so $|p(z)| \geq\left|a_{0}\right|-\left|a_{1} z+\cdots+a_{n} z^{n}\right|>0$.

(ii) Assume $p(z) \neq 0$ for all $z$ with $|z| \leq 1$. Then $p(0)=a_{0} \neq 0$ which leads to two cases: (1) if $a_{n}=0$ then obviously $\left|a_{0}\right|>\left|a_{n}\right|$; (2) if $a_{n} \neq 0$ we have $p(z)=a_{n}\left(z-z_{1}\right) \cdots\left(z-z_{n}\right)$ where $z_{n}$ are the zeros of $p$. But then $p(0)=a_{0}=$ $a_{n}\left(-z_{1}\right) \cdots\left(-z_{n}\right)$ and thus $\left|a_{0}\right|>\left|a_{n}\right|$ since $\left|z_{k}\right|>1$ for $k=1, \ldots, n$.

For each $p \in \mathcal{P}_{n}$ the reciprocal polynomial $p^{\#} \in \mathcal{P}_{n}$ is defined by

$$
p^{\#}(z)=z^{n} \cdot \overline{p(1 / \bar{z})}=\bar{a}_{n}+\bar{a}_{n-1} z+\cdots+\bar{a}_{1} z^{n-1}+\bar{a}_{0} z^{n} .
$$

Let us now define the Schur transform, $S_{n}: \mathcal{P}_{n} \rightarrow \mathcal{P}_{n-1}$ by

$$
\left(S_{n} p\right)(z)=\bar{a}_{0} p(z)-a_{n} p^{\#}(z)=\sum_{k=0}^{n-1}\left(\bar{a}_{0} a_{k}-a_{n} \bar{a}_{n-k}\right) z^{k}, \quad p \in \mathcal{P}_{n}
$$

We note a few simple facts pertaining to these objects.

First, $\left|p^{\#}\right|=|p|$ on $\mathbf{T}$. Moreover, every zero of $p$ on $\mathbf{T}$ is also a zero of $p^{\#}$ and is thus a zero of $S_{n} p$. Finally,

$$
\left(S_{n} p\right)(0)=\left|a_{0}\right|^{2}-\left|a_{n}\right|^{2} \in \mathbb{R} .
$$

We now construct a chain of polynomials $p_{0}, p_{1}, \ldots, p_{n}$ starting with $p_{0}=p$ and then taking successive Schur transforms,

$$
p_{1}=S_{n} p_{0}, \quad p_{2}=S_{n-1} p_{1}, \quad \ldots, \quad p_{n}=S_{1} p_{n-1} .
$$

The last polynomial $p_{n}$ is an element in $\mathcal{P}_{0}$ and thus is a constant.

Lemma 3.2 If $p_{k}$ has no zeros on the unit circle $\mathbf{T}$ and $p_{k+1}(0)>0$, then $p_{k}$ and $p_{k+1}$ have equally many zeros in $\overline{\mathbf{D}}$. 
Proof Let $p_{k}(z)=b_{0}+b_{1} z+\cdots+b_{n-k} z^{n-k}$. Then $p_{k+1}(z)=\bar{b}_{0} p_{k}-b_{n-k} p_{k}^{\#}$ and $p_{k+1}(0)=\left|b_{0}\right|^{2}-\left|b_{n-k}\right|^{2}>0$ so $\left|b_{0}\right|>\left|b_{n-k}\right|$. Since $p_{k}(z) \neq 0$ on $\mathbf{T}$ we have $\left|\bar{b}_{0} p_{k}\right|>\left|b_{n-k} p_{k}\right|=\left|b_{n-k} p_{k}^{\#}\right|$ on $\mathbf{T}$ and thus Rouché's theorem implies that $p_{k+1}$ and $p_{k}$ have equally many zeros in $\overline{\mathbf{D}}$.

We are now ready to formulate Schur-Cohn's test (e.g. [28]).

Theorem 3.1 A polynomial $p \in \mathcal{P}_{n}$ has no zeros in $\overline{\mathbf{D}}$ if and only if $p_{k}(0)>0$ for $k=1, \ldots, n$.

Proof Assume that $p=p_{0}$ has no zeros in $\overline{\mathbf{D}}$. By Lemma 3.1, $p_{1}(0)=\left|a_{0}\right|^{2}-\left|a_{n}\right|^{2}>$ 0 . Since $p_{0}$ has no zeros on $\mathbf{T}$, Lemma 3.2 implies that $p_{1}$ and $p_{0}$ have the same number of zeros in $\overline{\mathbf{D}}$, i.e., none. If $n \geq 2$ we can repeat the reasoning with $p_{1} \in \mathcal{P}_{n-1}$ instead of $p_{0}$ and deduce $p_{2}(0)>0$ and so on, and after a finite number of steps we finally get $p_{n}(0)>0$.

To prove the reverse implication, assume that $p_{k}(0)>0$ for $k=1, \ldots, n$. Then $p_{n}$ is a non-zero constant, and especially has no zeros on $\mathbf{T}$. Since $p_{n}=S_{1} p_{n-1}, p_{n-1}$ has no zeros on $\mathbf{T}$ and by Lemma 3.2, $p_{n-1}$ and $p_{n}$ has the same number of zeros in $|z| \leq 1$, i.e., none. We may now repeat this with $p_{n-1} \in \mathcal{P}_{1}$ instead of $p_{n}$ and deduce that $p_{n-2}$ has no zeros in $\overline{\mathbf{D}}$ and so on, and after a finite number of steps we finally get that $p=p_{0}$ has no zeros in $\overline{\mathbf{D}}$.

\section{Real Comprehensive Triangular Decomposition}

The method of real triangular decompositions, introduced recently in [7, §4], [5, §10] and [6], provides a suitable framework to deal with the the mapping problem for quadrature domains, in the form of systems such as (4), (5). These methods in turn make use of the idea of a Cylindrical Algebraic Decomposition [3, §5], for an overview of this and other applicable methods from real algebraic geometry see, for example, the book [3].

The objects we consider here are semi-algebraic sets, given a list of polynomial equations and inequalities in $\mathbf{R}\left[x_{1}, \ldots, x_{m}\right]$ a basic semi-algebraic set is the set of all points in $\mathbf{R}^{m}$ which simultaneously satisfy all these equations and inequalities, [3, $\S 3]$. In this section we will specifically consider semi-algebraic systems defined by polynomials in the polynomial ring

$$
\mathbb{Q}[c, x]=\mathbb{Q}\left[c_{1}, \ldots, c_{d}\right]\left[x_{1}, \ldots, x_{n}\right],
$$

where we think of the $c_{i}$ as parameters and the $x_{j}$ as variables.

More precisely, given polynomials $f_{1}, \ldots, f_{r}$ and $p_{1}, \ldots, p_{s}$ in $\mathbb{Q}[c, x]$, we define the semi-algebraic system $\left[f_{=0}, p_{>0}\right]$ to be the following set of equations and inequalities

$$
f_{1}(c, x)=0, \quad \ldots, \quad f_{r}(c, x)=0, \quad p_{1}(c, x)>0, \quad \ldots, \quad p_{s}(c, x)>0 .
$$

The set of real solutions ( $c, x) \in \mathbf{R}^{d} \times \mathbf{R}^{n}$ to (12) is called the (parameterized) semialgebraic set generated by the system, denoted by $\mathcal{S}\left(\left[f_{=0}, p_{>0}\right]\right)$. Moreover, for fixed 
$c \in \mathbf{R}^{d}$ we define the specialized semi-algebraic set $\mathcal{S}_{(c)}\left(\left[f_{=0}, p_{>0}\right]\right)$ as the set of points $x \in \mathbf{R}^{n}$ which satisfy the system (12) for the particular parameter-value $c$.

Now suppose that $\mathfrak{T}=\left\{\mathcal{T}_{1}, \ldots, \mathcal{T}_{\ell}\right\}$ is a collection of semi-algebraic systems in $\mathbb{Q}[c, x]$. We extend the definitions of semi-algebraic set and specialization to a parameter value $c$ by

$$
\mathcal{S}(\mathfrak{T})=\bigcup_{j=1}^{\ell} \mathcal{S}\left(\mathcal{T}_{j}\right) \subset \mathbf{R}^{d} \times \mathbf{R}^{n}, \quad \mathcal{S}_{(c)}(\mathfrak{T})=\bigcup_{j=1}^{\ell} \mathcal{S}_{(c)}\left(\mathcal{T}_{j}\right) \subset \mathbf{R}^{n}
$$

Moreover, given a semi-algebraic system $\mathcal{T}=\left[f=0, p_{>0}\right]$ we define the constructible set of $\mathcal{T}$ to be the set of complex solutions $(c, x) \in \mathbf{C}^{d} \times \mathbf{C}^{n}$ to the system of equations and inequalities

$$
f_{1}(c, x)=\ldots=f_{r}(c, x)=0, \quad p_{1}(c, x) \neq 0, \quad \ldots, \quad p_{s}(c, x) \neq 0 .
$$

We denote by $\mathbf{C S}(\mathcal{T})$ the set of solutions $(c, x) \in \mathbf{C}^{d} \times \mathbf{C}^{n}$ to (13); given $c \in \mathbf{C}^{d}$ we define the associated specialization $\mathbf{C S}_{(c)}(\mathcal{T})$ to be the set of points $x \in \mathbf{C}^{n}$ such that $(c, x) \in \mathbf{C S}(\mathcal{T})$.

If $\mathfrak{T}=\left\{\mathcal{T}_{1}, \ldots, T_{\ell}\right\}$ is a finite collection of semi-algebraic systems, it should now be obvious how to extend our definitions of constructible set $\left(\mathbf{C S}(\mathfrak{T})=\bigcup_{j} \mathbf{C S}\left(\mathcal{T}_{j}\right)\right)$ and specializations $\left(\mathbf{C} \mathcal{S}_{(c)}(\mathfrak{T})=\bigcup_{j} \mathbf{C S}_{(c)}\left(\mathcal{T}_{j}\right) \subset \mathbf{C}^{n}\right)$.

A semi-algebraic system $\mathcal{T}=\left[f_{=0}, p_{>0}\right]$ is called square-free if all polynomials $f_{j}$ and $p_{i}$ occurring in $\mathcal{T}$ are square-free. (A polynomial $g \in \mathbb{Q}[c, x]$ is square-free if it has no factor of the form $w^{2}$ where $w \in \mathbb{Q}[c, x]$ is non-constant.)

Definition 4.1 Let $\mathcal{W}=\left[f_{=0}, p_{>0}\right]$ be a semi-algebraic system defined by polynomials $f_{1}, \ldots, f_{r}$ and $p_{1}, \ldots, p_{s}$ in $\mathbb{Q}[c, x]$ and let $\mathcal{S}(\mathcal{W}) \subset \mathbf{R}^{d} \times \mathbf{R}^{n}$ be the associated semi-algebraic set.

A real comprehensive triangular decomposition $(R C T D)$ of $\mathcal{W}$ is a pair $\left(\mathfrak{C},\left\{\mathfrak{T}_{C} ; C \in\right.\right.$ $\mathfrak{C}\})$ where $\mathfrak{C}$ is a finite partition of $\mathbf{R}^{d}$ into non-empty semi-algebraic sets $C$ (called "cells") and for each $C \in \mathfrak{C}, \mathfrak{T}_{C}$ is a finite set of square-free semi-algebraic systems such that exactly one of the following holds:

(1) $\mathfrak{T}_{C}$ is empty so $\mathcal{S}\left(\mathfrak{T}_{C}\right)=\mathbf{R}^{d} \times \mathbf{R}^{n}$ and $\mathcal{S}_{(c)}\left(\mathfrak{T}_{C}\right)=\mathbf{R}^{n}$ for all $c \in \mathbf{R}^{d}$,

(2) The specialized constructible set $\mathbf{C} \mathcal{S}_{(c)}\left(\mathfrak{T}_{C}\right)$ is infinite for all $c \in C$,

(3) $\mathfrak{T}_{C}=\left\{\mathcal{T}_{1}, \ldots, \mathcal{T}_{\ell}\right\}$ is a finite set of semi-algebraic systems satisfying the following conditions:

- $\mathbf{C} \mathcal{S}_{(c)}\left(\mathfrak{T}_{C}\right)$ is finite and has fixed cardinality for all $c \in C$,

- the specialized semi-algebraic sets $\mathcal{S}_{(c)}\left(\mathcal{T}_{j}\right)$ are finite and non-empty for all $j$ and further for a fixed $\mathcal{T}_{j}$ the specialized semi-algebraic set $\mathcal{S}_{(c)}\left(\mathcal{T}_{j}\right)$ has fixed cardinality for all $c \in C$,

- $\mathcal{S}_{(c)}(\mathcal{W})=\bigsqcup_{j=1}^{\ell} \mathcal{S}_{(c)}\left(\mathcal{T}_{j}\right)$ for all $c \in C$.

The following proposition summarizes the results about RCTD's that we will apply in the sequel. 
Fig. 4 The cells $\mathfrak{C}$ from the RCTD in Example 4.1

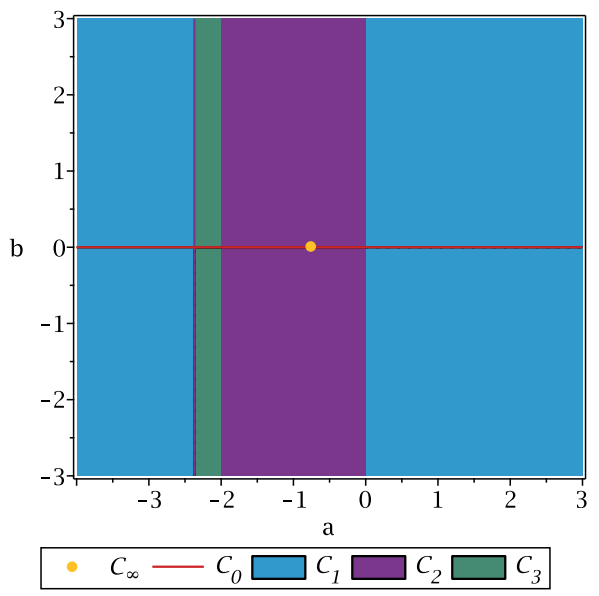

Proposition 4.1 (\$10 of [5]) Let $f_{1}, \ldots, f_{r}$ and $p_{1}, \ldots, p_{s}$ be polynomials in the polynomial ring $\mathbb{Q}[c, x]$ defining a semi-algebraic system $\mathcal{W}=\left[f_{=0}, p_{>0}\right]$. Then a real comprehensive triangular decomposition of $\mathcal{W}$ exists and may be computed by an explicit algorithm which is guaranteed to terminate in finite time. This algorithm is implemented in the RegularChains Maple package [31].

Example 4.1 Define $f=a \cdot y^{2}+b \cdot x+3$ and $g=b \cdot x y-y+2$ where $x, y$ denote real variables and $a, b$ denote real parameters. Consider the semi-algebraic system

$$
\mathcal{W}=[f=0, g=0, y \leq 1]
$$

Using the RegularChains package, the parameter space $\mathbf{R}^{2}$ is partitioned into five cells $\mathfrak{C}=\left\{C_{0}, C_{1}, C_{2}, C_{3}, C_{\infty}\right\}$ where

$$
\begin{aligned}
& C_{\infty}=\left\{\left(-\frac{3}{4}, 0\right)\right\}, \quad C_{0}=\left\{(a, b) \in \mathbf{R}^{2} ; a \neq-\frac{3}{4}, b=0\right\}, \\
& C_{1}=\left\{(a, b) \in \mathbf{R}^{2} ; a<-\frac{64}{27}, b \neq 0\right\} \bigsqcup\left\{(a, b) \in \mathbf{R}^{2} \mid a \geq 0, b \neq 0\right\}, \\
& C_{2}=\left\{(a, b) \in \mathbf{R}^{2} ; a=-\frac{64}{27}, b \neq 0\right\} \bigsqcup\left\{(a, b) \in \mathbf{R}^{2} \mid-2<a<0, b \neq 0\right\}, \\
& C_{3}=\left\{(a, b) \in \mathbf{R}^{2} ;-\frac{64}{27}<a \leq-2, b \neq 0\right\} .
\end{aligned}
$$

These cells are illustrated in Fig. 4.

It is not hard to show that the specialized semi-algebraic set $\mathcal{S}_{(a, b)}(\mathcal{W})$ consists of $j$ points for all $(a, b) \in C_{j}$ where $j \in\{0,1,2,3\}$; for the cell $C_{\infty}$ the RCTD guarantees only that the specialized constructible set associated to the parameter choice $C_{\infty}$ has infinitely many points (a corresponding specialized semi-algebraic set may be infinite, empty, or finite). Hence $\mathfrak{C}$, along with the associated semi-algebraic systems for each parameter cell, gives a RCTD of $\mathcal{W}$. 
In more detail; consider the cells $C_{0}$ and $C_{\infty}$. The disjoint union of these cells is the line $b=0$ (i.e. the a-axis in Fig. 4). Along this line the semi-algebraic system $\mathcal{W}$ simplifies to

$$
a \cdot y^{2}+3=2-y=0, \quad y \leq 1
$$

Solving for $y$ we obtain $y=\sqrt{-3 / a}$ and $y=2$, so $f=g=0$ has a real solution if and only if $a=-3 / 4$, so $\{(a, b)\}=C_{\infty}$. The corresponding specialized constructible set is

$$
\left\{(x, y) \in \mathbf{C}^{2} ;(y-2)(y+2)=(y+2)=0, \quad y \neq 1\right\}=\{(x, 2) ; x \in \mathbf{C}\},
$$

which is infinite. However since $y \leq 1$ is never satisfied the semi-algebraic set $\mathcal{S}\left(\mathcal{I}_{C_{\infty}}\right)$ is empty. On the other hand in the cell $C_{0}$ it is clear that the semi-algebraic system (14) has no real solutions, since $a \neq \frac{-3}{4}$ when $(a, b) \in C_{0}$.

Now consider the subset $P$ of the cell $C_{2}$ consisting of all $(a, b)$ where $a=-64 / 27$ and $b \neq 0$. For $(a, b) \in P$ the system $\mathcal{W}$ simplifies to

$$
\left[x=\frac{7}{3 b}, y=\frac{-3}{2}, y \leq 1\right] \text { or }\left[x=\frac{-5}{3 b}, y=\frac{3}{4}, y \leq 1\right] \text {. }
$$

Clearly the system (15) has precisely two solutions for any $b \neq 0$, namely $(x, y)=$ $(7 /(3 b),-3 / 2)$ and $(x, y)=(-5 /(3 b), 3 / 4)$. Similarly all other choices of $(a, b) \in$ $C_{2}$ yield associated specialized semi-algebraic sets with exactly two points.

\section{Some Computational Results}

In this section we apply the method of real comprehensive triangular decompositions to obtain (new) proofs of uniqueness for certain families of quadrature domains.

\subsection{Aharonov-Shapiro 1976}

In this subsection, we shall give an alternative proof of a theorem of Aharonov and Shapiro from [2], which states that a solid quadrature domain obeying a quadrature identity of the form

$$
\int_{\Omega} f d A=M_{0} f(0)+M_{1} f^{\prime}(0), \quad f \in A L^{1}(\Omega)
$$

is unique. Here $M_{0}$ is the area of $\Omega$, so necessarily $M_{0}>0$. The constant $M_{1}$ is allowed to be an arbitrary complex number.

Recall that the computations in Example 2.2 show that a normalized mapping function $\varphi: \mathbf{D} \rightarrow \Omega$ is necessarily a polynomial of degree at most 2 , which solves 
the system

$$
\left\{\begin{array}{l}
w_{1}^{3} \bar{w}_{1}=M_{0} w_{1}^{2}-M_{1} w_{2} \\
w_{1}^{2} \bar{w}_{2}=2 M_{1}
\end{array} \quad, \quad w_{1}:=\varphi^{\prime}(0)>0, \quad w_{2}:=\varphi^{\prime \prime}(0)\right.
$$

It remains to show that this system gives rise to a unique univalent solution. For this, we write

$$
M_{1}:=m_{1}+i n_{1}, \quad w_{2}:=u_{2}+i v_{2}
$$

We now obtain the following semi-algebraic system, which is equivalent to (17),

$$
(\mathcal{W}) \quad\left\{\begin{array}{l}
w_{1}^{4}=M_{0} w_{1}^{2}-\left(m_{1} u_{2}-n_{1} v_{2}\right) \\
0=m_{1} v_{2}+n_{1} u_{2} \\
w_{1}^{2} u_{2}=2 m_{1} \\
-w_{1}^{2} v_{2}=2 n_{1} \\
M_{0}>0 \\
w_{1}>0 \\
\left(w_{1}^{2} M_{0}-\left(m_{1} u_{2}-n_{1} v_{2}\right)\right)^{2}-4 w_{1}^{2}\left(m_{1}^{2}+n_{1}^{2}\right) \geq 0
\end{array}\right.
$$

We must verify that the system $\mathcal{W}$ gives rise to at most one univalent solution for all relevant choices of quadrature data. For this, we first recognize that the last condition in $\mathcal{W}$ is just the Schur-Cohn constraint (see Theorem 3.1), which ensures that $\varphi^{\prime}(z) \neq 0$ in $\mathbf{D}$. In general this is only necessary for univalence but for polynomials of degree two it is also sufficient. We shall treat $c=\left(m_{1}, n_{1}, M_{0}\right)$ as parameters. Computing a real comprehensive triangular decomposition of the system $\mathcal{W}$, using the RegularChains library, we obtain a partition of the parameter space $\mathbf{R}^{3}$ into two cells $C_{0}, C_{1}$ having the following properties.

All points in the cell $C_{0}$ are such that $M_{0} \leq 0$; hence no point of $C_{0}$ can correspond to a quadrature domain. With $\omega:=\sqrt[3]{\left(27 m_{1}^{2}+27 n_{1}^{2}\right) / 2}$, the cell $C_{1}$ is expressed as the disjoint union of the following six subsets of $\mathbf{R}^{3}$,

(i)

$$
\left\{\begin{array}{l}
M_{0}=\omega \\
m_{1}=m_{1} \\
n_{1}<0
\end{array}, \quad\left\{\begin{array}{l}
M_{0}=\omega \\
m_{1}=m_{1} \\
n_{1}>0
\end{array}\right.\right.
$$

(ii)

$$
\left\{\begin{array}{l}
M_{0}=\omega \\
m_{1}<0 \\
n_{1}=0
\end{array}\right.
$$


(iii)

$$
\left\{\begin{array}{l}
M_{0}=\omega \\
m_{1}>0 \\
n_{1}=0
\end{array}\right.
$$

(iv)

$$
\left\{\begin{array}{l}
M_{0}>\omega \\
m_{1}=m_{1} \\
n_{1}<0
\end{array}, \quad\left\{\begin{array}{l}
M_{0}>\omega \\
m_{1}=m_{1} \\
n_{1}>0
\end{array}\right.\right.
$$

(v)

$$
\left\{\begin{array}{l}
M_{0}>\omega \\
m_{1}<0 \\
n_{1}=0
\end{array}, \quad\left\{\begin{array}{l}
M_{0}>\omega \\
m_{1}>0 \\
n_{1}=0
\end{array}\right.\right.
$$

(vi)

$$
\left\{\begin{array}{l}
M_{0}>0 \\
m_{1}=0 \\
n_{1}=0
\end{array} .\right.
$$

For $\left(M_{0}, m_{1}, n_{1}\right)$ in each of these sets, we now prove that the system (17) has a unique solution $\left(w_{1}, w_{2}\right)=\left(w_{1}, u_{2}+i v_{2}\right)$ with $w_{1}>0$. Indeed, straightforward calculations show that in each of the parameter-domains, (i)-(vi) the semi-algebraic system $\mathcal{W}$ simplifies to, respectively,

(i)

$$
\left\{\begin{array}{l}
v_{2} w_{1}^{2}+2 n_{1}=0 \\
n_{1} u_{2}+v_{2} m_{1}=0 \\
\left(9 m_{1}^{2}+9 n_{1}^{2}\right) v_{2}+2 M_{0}^{2} n_{1}=0 \\
w_{1}>0
\end{array}\right.
$$

(ii)

$$
\left\{\begin{array}{l}
2 u_{2} w_{1}-u_{2}^{2}+2 M_{0}=0 \\
9 m_{1} u_{2}-2 M_{0}^{2}=0 \\
v_{2}=0
\end{array}\right.
$$


(iii)

$$
\left\{\begin{array}{l}
2 u_{2} w_{1}+u_{2}^{2}-2 M_{0}=0 \\
9 m_{1} u_{2}-2 M_{0}^{2}=0 \\
v_{2}=0
\end{array}\right.
$$

(iv)

$$
\left\{\begin{array}{l}
v_{2} w_{1}^{2}+2 n_{1}=0 \\
n_{1} u_{2}+v_{2} m_{1}=0 \\
\left(m_{1}^{2}+n_{1}^{2}\right) v_{3}^{2}-2 M_{0} n_{1}^{2} v_{2}-4 n_{1}^{3}=0 \\
w_{1}>0 \\
-v_{2}^{2} M_{0} m_{1}^{2} n_{1}^{2}-v_{2}^{2} M_{0} n_{1}^{4}+2 M_{0}^{2} n_{1}^{4}+6 v_{2} m_{1}^{2} n_{1}^{3}+6 v_{2} n_{1}^{5}>0
\end{array}\right.
$$

(

$$
\left\{\begin{array}{l}
u_{2} w_{1}^{2}-2 m_{1}=0 \\
u_{2}^{3}-2 M_{0} u_{2}+4 m_{1}=0 \\
v_{2}=0 \\
w_{1}>0 \\
-M_{0} m_{1}^{2} u_{2}^{2}+2 M_{0}^{2} m_{1}^{2}-6 m_{1}^{3} u_{2}-4 m_{1} n_{1}^{2} u_{2}>0
\end{array}\right.
$$

(vi)

$$
\left\{\begin{array}{l}
w_{1}^{2}-M_{0}=0 \\
u_{2}=0 \\
v_{2}=0 \\
w_{1}>0
\end{array}\right.
$$

In each case (i)-(vi), we have a unique solution $\left(w_{1}, w_{2}\right)=\left(w_{1}, u_{2}+i v_{2}\right)$ where $w_{1}>0$, which concludes our automated proof of uniqueness for solid q.d.'s obeying (16).

Remark The uniqueness problem for quadrature domains of order 2 has been completely settled, see [19, Cor. 10.1]. More precisely, counting also non-simply connected domains and combining with results in [20], a given point functional $\mu$ of order 2 can give rise to at most two quadrature domains: one simply connected $\Omega$, and possibly another one of the form $\Omega \backslash\{a\}$ where $a$ is a "special point", i.e., $S(a)=\bar{a}$ where $S$ is the Schwarz function. The term "special point" is due to Shapiro in [39], see Theorem 2.9 and the discussion that precedes it.

These methods can also be applied to polynomials of higher degree. (This topic is being considered as part of an ongoing work by the two of the authors.) 


\subsection{Symmetric Smash Sums}

Consider a domain $\Omega$ that satisfies

$$
\int_{\Omega} f d A=c\left(f\left(a_{0}\right)+\cdots+f\left(a_{n-1}\right)\right), \quad a_{j}=a e^{2 \pi i j / n}, f \in A L^{1}(\Omega),
$$

where $c>0$ and $a<0$ are constants and $n$ is a positive integer.

A domain satisfying (18) may be constructed as a potential theoretic sum (or "smash sum") of discs $D\left(a_{j} ; \sqrt{c}\right)$, where excess mass coming from overlapping discs is swept out using the process of partial balayage. When $n=2$ this process gives rise to the well-known Neumann's oval.

For $n \geq 3$ one can surmise that a (connected) domain satisfying (18) should be either simply connected or doubly connected, depending on whether or not $0 \in \Omega$. The doubly connected case has already been treated in [43, Section 5.8] and in [9]. (See Subsect. 7.3 for further remarks in this connection.)

In this subsection, we shall focus on the simply connected case and show how uniqueness of the quadrature domain follows using our general algebraic scheme. (In this particular case, there are, of course, alternative ways to see this, e.g. by Novikov's theorem, Theorem 1.1, (i).) form

Due to the $\mathbb{Z}_{n}$ symmetry of (18), the conformal map $\varphi: \mathbf{D} \rightarrow \Omega$ attains the simple

$$
\varphi(z)=\sum_{j=0}^{n-1} \frac{c_{j}}{\bar{w}_{j}} \frac{z}{1-\bar{\lambda}_{j} z}=\frac{c}{w} \sum_{j=0}^{n-1} \frac{z}{1-\exp \left(\frac{-2 \pi i}{n} j\right) \lambda z}=\frac{c n}{w} \frac{z}{1-\lambda^{n} z^{n}}
$$

where $w_{j}=\varphi^{\prime}\left(\lambda_{j}\right)=w>0,-1<\lambda=\varphi^{-1}(a)<0$. This gives

$$
\varphi^{\prime}(z)=\frac{c n}{w} \frac{1+(n-1) \lambda^{n} z^{n}}{\left(1-\lambda^{n} z^{n}\right)^{2}} .
$$

The two unknowns, $\lambda$ and $w(-1<\lambda<0$ and $w>0)$ satisfy

$$
\left\{\begin{array} { l } 
{ a = \frac { c n } { w } \frac { \lambda } { 1 - \lambda ^ { 2 n } } } \\
{ w = \frac { c n } { w } \frac { 1 + ( n - 1 ) \lambda ^ { 2 n } } { ( 1 - \lambda ^ { 2 n } ) ^ { 2 } } }
\end{array} \Longleftrightarrow \left\{\begin{array}{l}
a w\left(1-\lambda^{2 n}\right)=n c \lambda \\
w^{2}\left(\lambda^{4 n}-2 \lambda^{2 n}+1\right)=n c\left((n-1) \lambda^{2 n}+1\right) .
\end{array}\right.\right.
$$

We only need to check solutions $\varphi$ which are locally univalent. From (20) (or from Schur-Cohn's test) one may easily deduce that local univalence holds if $1 \geq(n-1) \lambda^{n}$. 
Taking this into consideration we obtain the following semi-algebraic system

$$
\left\{\begin{array}{l}
a w\left(1-\lambda^{2 n}\right)=n c \lambda \\
w^{2}\left(\lambda^{4 n}-2 \lambda^{2 n}+1\right)=n c\left((n-1) \lambda^{2 n}+1\right) \\
c>0 \\
a<0 \\
w>0 \\
-1<\lambda<0 \\
1 \geq(n-1)^{2} \lambda^{2 n} .
\end{array}\right.
$$

When setting up a real comprehensive triangular decomposition, we cannot treat $n$ as a parameter but have to use a fixed value of $n$ (since if $n$ is treated as a parameter the system (21) is no longer semi-algebraic). Below we show the results for $n=3$ but the corresponding decompositions may be obtained for up to $n=10$ without any runtime issues.

Thus let $\mathcal{W}$ be the semi-algebraic system (21) with $n=3$ fixed. The associated semi-algebraic set $\mathcal{S}(\mathcal{W})$ is contained in $\mathbf{R}^{2} \times \mathbf{R}^{2}$. We treat $(a, c) \in \mathbf{R}^{2}$ as parameters and consider the specialized semi-algebraic set $\mathcal{S}_{(a, c)}(\mathcal{W}) \subset \mathbf{R}^{2}$. Using an RCTD, we have found that the parameter space $\mathbf{R}^{2}$ is divided into two cells $C_{0}, C_{1}$ such that for all $(a, c) \in C_{1}$ the system $\mathcal{W}$ has precisely one real solution and for all $(a, c) \in C_{0}$ the system $\mathcal{W}$ has no real solutions. The cell $C_{1}$ is made up of the three regions defined in (i), (i), and (iii) below.

(i)

$$
\left\{\begin{array}{l}
c=\frac{a^{2}}{2^{1 / 3}} \\
a<0
\end{array}\right.
$$

(ii)

$$
\left\{\begin{array}{l}
\frac{a^{2}}{2^{1 / 3}}<c<a^{2} \\
a<0
\end{array},\left\{\begin{array}{l}
a^{2}<c \\
a<0
\end{array}\right.\right.
$$

(iii)

$$
\left\{\begin{array}{l}
c=a^{2} \\
a<0
\end{array} .\right.
$$

In each of the domains above, the semi-algebraic system (21) simplifies as follows

(i)

$$
\left\{\begin{array}{l}
\left(\lambda^{6}-1\right) a w+3 \lambda c=0 \\
a^{2} \lambda+c=0
\end{array}\right.
$$


(ii)

$$
\left\{\begin{array}{l}
\left(\lambda^{6}-1\right) a w+3 \lambda c=0 \\
2 a^{2} \lambda^{6}-3 c \lambda^{2}+a^{2}=0 \\
w>0 \\
-4 \lambda^{6}+1>0 \\
-\lambda>0 \\
\lambda+1>0
\end{array}\right.
$$

(iii)

$$
\left\{\begin{array}{l}
\left(\lambda^{2}-1\right) w+2 \lambda a=0 \\
2 \lambda^{4}+2 \lambda^{2}-1=0 \\
w>0 \\
-4 \lambda^{6}+1>0 \\
-\lambda>0 \\
\lambda+1>0
\end{array}\right.
$$

Since for all choices of the parameters in the cell $C_{1}$ we know from the definition of the RCTD that the number of real solutions is fixed, picking a particular $a$ and $c$ in $C_{1}$ it is easy to verify that the systems above have unique real solutions which correspond to a quadrature domain. Similarly, one can pick a choice of parameters in $C_{0}$ to verify that the system (21) has no real solutions for any parameter choice in $C_{0}$. In particular, we have shown that a quadrature domain obeying a quadrature identity (18) is uniquely determined. However, we cannot yet be sure that each solution to the system (21) defines a univalent map since we have only required local univalence. The following proposition shows that this is in fact the case.

Proposition 5.1 The mapping $\varphi$ in (19), $(-1<\lambda<0)$, is univalent if and only if

$$
1 \geq(n-1)^{2} \lambda^{2 n}
$$

Proof The necessity is obvious since the strict inequality $1>(n-1)^{2} \lambda^{2 n}$ is just the Schur-Cohn condition for $\varphi^{\prime}$ to have no zeros in $\overline{\mathbf{D}}$.

To prove sufficiency we shall prove that (22) implies that $\varphi$ is starlike, and in particular univalent. Recall ( [34, Thm. 2.5]) that starlikeness is equivalent to that $\operatorname{Re}\left(z \varphi^{\prime}(z) / \varphi(z)\right)>0$ for all $z \in \mathbf{D}$. Using the Cauchy-Riemann equations one finds that this is equivalent to

$$
\frac{d}{d r}|\varphi(z)|^{2}>0, \quad z=r e^{i \theta} \in \mathbf{D}
$$


(Cf. [16]). Using Eq. (19) we find that $\frac{d}{d r}|\varphi(z)|^{2}$ is a positive multiple of

$$
2 r\left(\lambda^{2 n} r^{2 n}(1-n)-\lambda^{n} r^{n}(2-n) \cos (n \theta)+1\right)
$$

This is a quadratic polynomial in $\lambda^{n}$ which is clearly positive when $\lambda=0$. We need to prove that it remains positive for all $r$ with $0 \leq r<1$ if $\lambda<0$ and (22) holds. We may assume that $r=1$ and $\cos (n \theta)= \pm 1$. For odd $n$, the non-negativity of (23) then means

$$
\lambda^{2 n}(1-n)-\lambda^{n}(2-n)+1 \geq 0
$$

while for even $n$, it means

$$
\lambda^{2 n}(1-n)+\lambda^{n}(2-n)+1 \geq 0
$$

By assumption $\lambda<0$, so the inequalities (24), (25) amount to $\lambda^{n}(n-1) \leq 1$ and $-1 \geq \lambda^{n}(n-1)$ respectively. This is equivalent to $1 \geq \lambda^{2 n}(n-1)^{2}$ and proves that $\varphi$ is starlike if and only if (22) holds.

\section{Three Examples of Uniqueness}

In this section we give several further examples of uniqueness of quadrature domains based on real comprehensive triangular decomposition and other computational methods from algebraic geometry (i.e. Gröbner basis computations).

Example 6.1 Let $\Omega$ be a quadrature domain satisfying

$$
\int_{\Omega} f d A=f(0)+f\left(\frac{265}{153}+i\right)+f\left(\frac{265}{153}-i\right), \quad f \in A L^{1}(\Omega)
$$

the nodes are chosen such that the union $D(0,1) \cup D(265 / 153+i, 1) \cup D(265 / 153-$ $i, 1$ ) is just barely simply connected (this is guaranteed by $265 / 153<\sqrt{3}$ ), see Fig. 5.

To find the mapping $\varphi: \mathbf{D} \rightarrow \Omega$ we need to solve the six complex equations $a_{i}=\varphi\left(\lambda_{i}\right), w_{i}=\varphi^{\prime}\left(\lambda_{i}\right), i=1,2,3$, or equivalently 12 real equations. Without loss of generality we pick $\lambda_{1}=0=a_{0}$ and $w_{1}>0$, moreover, using the mirror symmetry in (26) we get $\lambda_{2}=\bar{\lambda}_{3}$ and $w_{2}=\bar{w}_{3}$. This means that in total we only have to solve for five real variables. The equations are, of course, rational, meaning that in order to calculate the Gröbner basis we need to clear denominators and algebraically remove the new roots introduced by this. These new roots correspond to poles of the original rational system, and hence are not of interest. The defining equations without roots at the poles are obtained by saturating the resulting ideal by the product of the equations from the denominators.

A Gröbner basis calculation shows that there is a unique solid quadrature domain obeying (26), depicted in Fig. 5. To be explicit, we can write $\left(\lambda_{1}, \lambda_{2}, \lambda_{3}\right)=(0, l+$ 


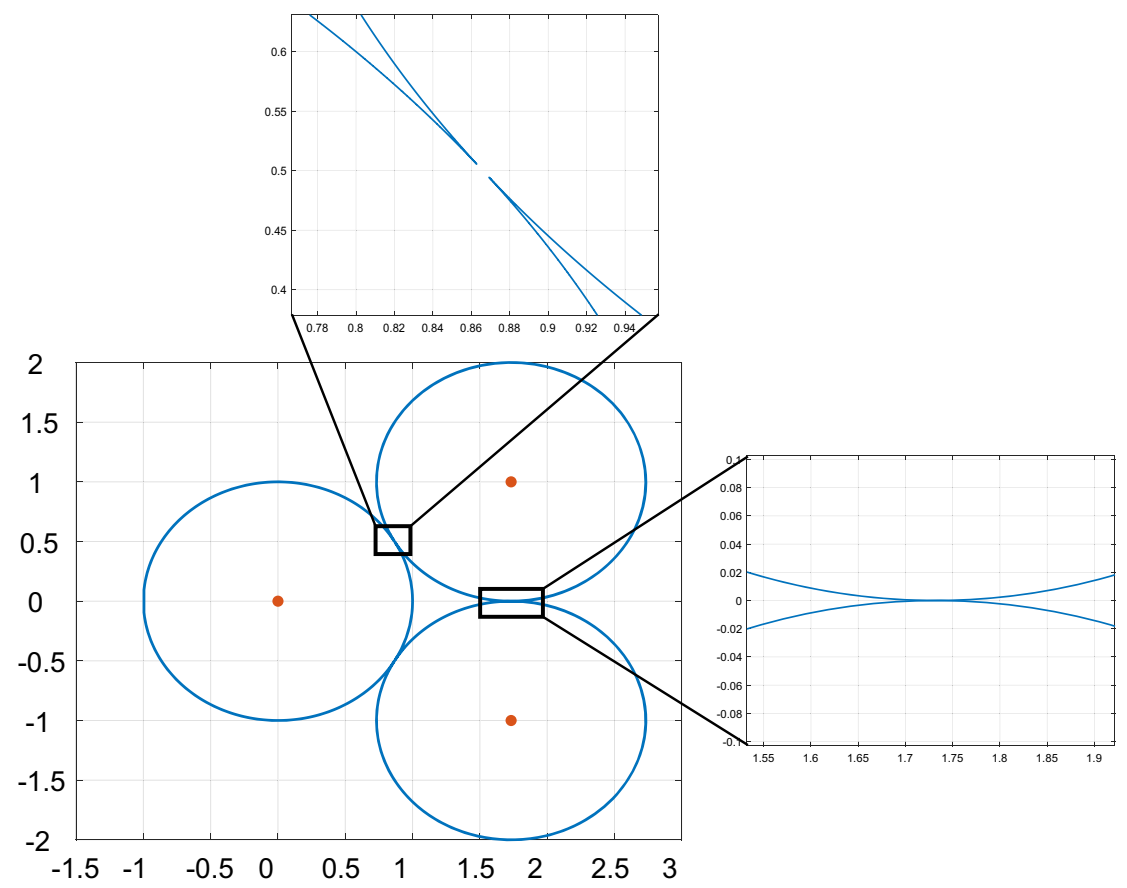

Fig. 5 The quadrature domain defined by Eq. (26). (A close inspection shows that the domain is in fact simply connected.)

$i p, l-i p)$ and $\left(w_{1}, w_{2}, w_{3}\right)=\left(x_{1}, x_{2}+i y_{2}, x_{2}-i y_{2}\right)$, where

$$
\begin{aligned}
l & =0.866022320861578 \ldots, \\
p & =0.499994659802733 \ldots, \\
x_{1} & =1.00001067993828 \ldots, \\
x_{2} & =93633.9999439279 \ldots, \\
y_{2} & =0.866068567766777 \ldots .
\end{aligned}
$$

Note that the values $l, p, x_{1}, x_{2}, y_{2}$ above can be obtained as exact expressions in a field extension of $\mathbb{Q}$ from the result of our Gröbner basis computation, however we opt to give the numerical approximations here for simplicity.

Example 6.2 Let $c_{1}>0$ and let $\Omega$ be a quadrature domain satisfying

$\int_{\Omega} f d A=c_{1}\left[f(-2)+f\left(-2 e^{2 \pi i / 3}\right)+f\left(-2 e^{4 \pi i / 3}\right)\right]+f(0), \quad f \in A L^{1}(\Omega)$. 
This is similar to a $\mathbb{Z}_{3}$-symmetric domain in Subsect. 5.2, but with an additional node at the origin. Assuming $\Omega$ is simply connected, the mapping $\varphi: \mathbf{D} \rightarrow \Omega$ is given by

$$
\varphi(z)=\frac{3 c_{1}}{w_{1}} \frac{z}{1-\lambda^{3} z^{3}}+\frac{c_{2}}{w_{2}} z
$$

where $\varphi(\lambda)=-2=a, \varphi^{\prime}(\lambda)=w_{1}, \varphi^{\prime}(0)=w_{2}$ and $c_{2}=1$. Clearing denominators leads to the semi-algebraic system

$$
\left\{\begin{array}{l}
3 c_{1} \lambda\left(\lambda^{6}-1-w_{2}\right)=-2 w_{1} w_{2}\left(\lambda^{6}-1\right) \\
3 c_{1}\left(\lambda^{1} 2-2 \lambda^{6}\left(1-w_{2}\right)+1+w_{2}\right)=w_{1}^{2} w_{2}\left(\lambda^{6}-1\right) \\
3 c_{1}\left(1+w_{2}\right)=w_{1} w_{2}^{2} \\
c_{1}>0 \\
-1<\lambda<0 \\
w_{1}>0, w_{2}>0
\end{array}\right.
$$

with variables $w_{1}, w_{2}, \lambda$ and parameter $c_{1}$. Let $\mathcal{W}$ denote the semi-algebraic system (27). Computing a triangular decomposition yields two cells: $C_{0}=\left\{c_{1} \in \mathbf{R} \mid c_{1} \leq 1\right\}$ and $C_{1}=\left\{c_{1} \in \mathbf{R} \mid c_{1}>1\right\}$ in the parameter space $\mathbf{R}$ (with coordinate $c_{1}$ ). For each $c_{1} \in C_{0}$ the system $\mathcal{W}$ has no real solutions while for each $c_{1} \in C_{1}$ the system $\mathcal{W}$ has exactly one real solution $\left(w_{1}, w_{2}, \lambda\right) \in \mathbf{R}^{3}$; note that $\mathbf{R}=C_{0} \sqcup C_{1}$.

Observe that to obtain a solution, we must have $c_{1}>1$ in order that $\Omega$ be simply connected (see Fig. 6(c)). If we interpret $c_{1}$ as time, we may think of the quadrature domain $\Omega\left(c_{1}\right)$ as the result of a Hele-Shaw evolution, starting from $\Omega(0)=D(0,1)$, after injecting fluid through the nodes $-2,-2 e^{2 \pi i / 3},-2 e^{4 \pi i / 3}$ at constant rate 1 , for $c_{1}$ units of time (see Subsect. 7.2 below for more about this). The evolution is depicted in Fig. 6.

Example 6.3 Let $\Omega$ be a quadrature domain satisfying

$$
\int_{\Omega} f d A=-\frac{1}{2}(f(a)+f(-a))+2 f(0), \quad f \in A L^{1}(\Omega) .
$$

This is a $\mathbb{Z}_{n}$-symmetric domain with $n=2$ as in Subsect. 5.2 but with negative weights and an additional node at the origin with strength two. The mapping $\varphi: \mathbf{D} \rightarrow \Omega$ then satisfies

$$
\varphi(z)=\frac{2 c_{1}}{w_{1}} \frac{z}{1-\lambda^{2} z^{2}}+\frac{c_{2}}{w_{2}} z
$$

where $\varphi(\lambda)=a, w_{1}=\varphi^{\prime}(\lambda), w_{2}=\varphi^{\prime}(0), c_{1}=-1 / 2$ and $c_{2}=2$. To obtain unique solutions for this system we have to impose Schur-Cohn constraints; this is as easily done for arbitrary $n$ as for $n=2$ so we do it for arbitrary $n$. 

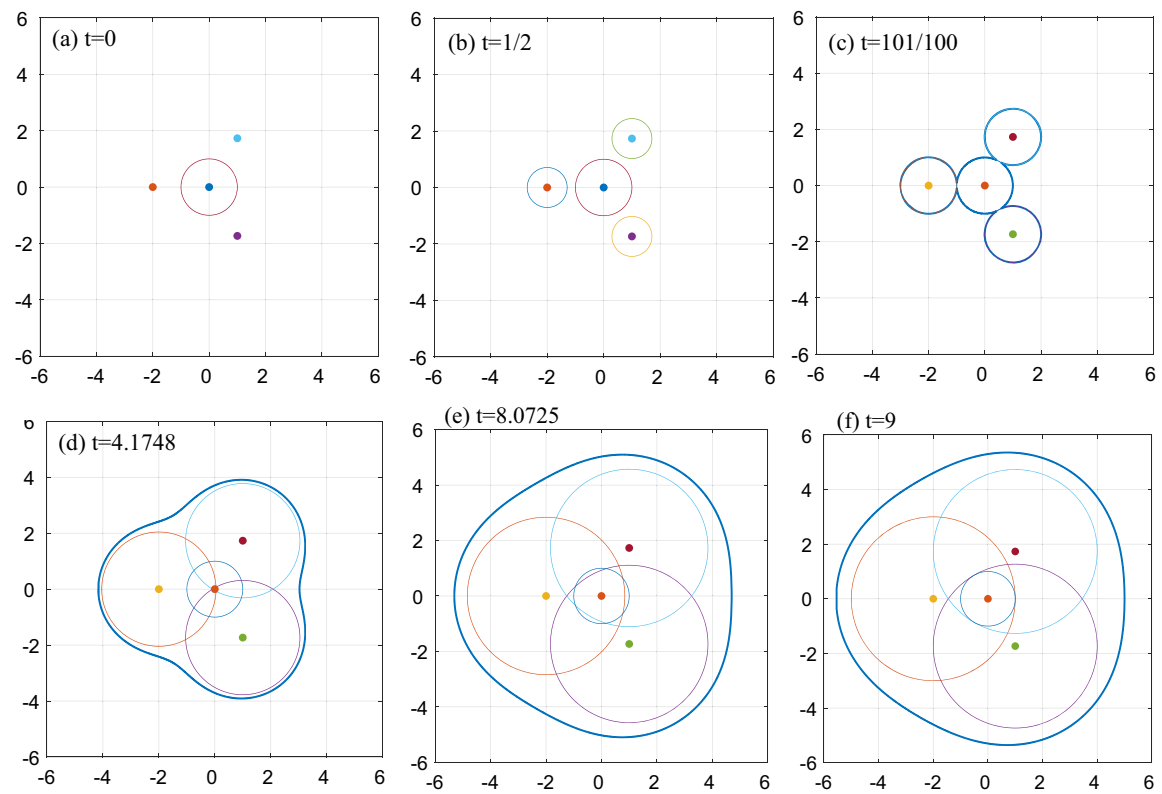

Fig. 6 Hele-Shaw growth of the unit disc $\mathbf{D}$ under injection at constant rate 1 at three nodes $-2,-2 e^{2 \pi i / 3},-2 e^{4 \pi i / 3}$. (a) and (b) lie in the cell $C_{0}$ of the RCTD; (a) is the initial domain D (time $t=0$ ) with the three injection points plotted, (b) corresponds to $t=1 / 2$ and is disconnected. In (c), $t=c_{1}>1$ so the domain is simply connected and lies in cell $C_{1}$ as do (d), (e), and (f)

Set $p(z)=b_{0}+b_{1} z^{n}+b_{2} z^{2 n}$ where $b_{0}=c_{1} w_{2} n, b_{1}=\lambda^{n}\left(c_{1} w_{2} n(n-1)-2 c_{2} w_{2}\right)$ and $b_{2}=c_{2} w_{1} \lambda^{2 n}$. The derivative of the mapping $\varphi$ is given by

$$
\varphi^{\prime}(z)=\frac{c_{1} n}{w_{1}} \frac{1+(n-1) \lambda^{n} z^{n}}{\left(1-\lambda^{n} z^{n}\right)^{2}}+\frac{c_{2}}{w_{2}}=\frac{p(z)}{w_{1} w_{2}\left(1-\lambda^{n} z^{n}\right)^{2}} .
$$

When $n=2$ we have that $b_{0}=4 c_{1} w_{2}, b_{1}=\lambda^{2}\left(2 c_{1} w_{2}-2 c_{2} w_{2}\right)$ and $b_{2}=c_{2} w_{1} \lambda^{4}$. It follows that the Schur-Cohn constraints for the $n=2$ case are given by

$$
\left\{\begin{array}{l}
b_{0}^{2}-b_{2}^{2} \geq 0 \\
\left(b_{0}^{2}-b_{2}^{2}\right)^{2}-\left(b_{0} b_{1}-b_{1} b_{2}\right)^{2} \geq 0
\end{array}\right.
$$

where we allow for equality to include the case where $\partial \Omega$ has cusps, see Fig. 7. Clearing denominators in the equations $\varphi(\lambda)=a, w_{1}=\varphi^{\prime}(\lambda), w_{2}=\varphi^{\prime}(0)$, recalling that $c_{1}=-1 / 2, c_{2}=2$, and adding the Schur-Cohn constraints we obtain the semi- 
Fig. 7 The unique solid quadrature domain satisfying (28) for the parameter-value $a=\vartheta \in C_{1}$

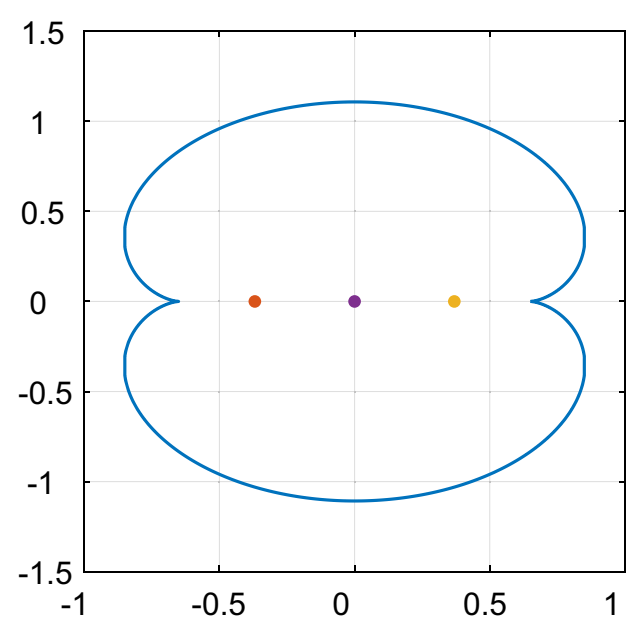

algebraic system

$$
\left\{\begin{array}{l}
\lambda\left(2 \lambda^{4} w_{1}-2 w_{1}+w_{2}\right)=a w_{1} w_{2}\left(\lambda^{4}-1\right) \\
w_{1}^{2} w_{2}\left(\lambda^{4}-1\right)^{2}=\varphi^{\prime}(\lambda)=\left(2 \lambda^{8}-4 \lambda^{4}+2\right) w_{1}-\lambda^{4} w_{2}-w_{2} \\
w_{1} w_{2}^{2}=2 w_{1}-w_{2} \\
b_{0}^{2}-b_{2}^{2} \geq 0 \\
\left(b_{0}^{2}-b_{2}^{2}\right)^{2}-\left(b_{0} b_{1}-b_{1} b_{2}\right)^{2} \geq 0 \\
a<0 \\
-1<\lambda<0 \\
w_{1}, w_{2}>0
\end{array}\right.
$$

where we treat $a$ as a parameter and $\left(w_{1}, w_{2}, \lambda\right)$ as variables (note $c_{1}, c_{2}$ are fixed). Let $\mathcal{W}$ denote the semi-algebraic system (29). Let $\tau=27 \sqrt{3}+54$ and set

$\vartheta=-\frac{1}{2} \sqrt{\frac{\tau^{2 / 3}+4 \tau^{1 / 3}+9}{\tau^{1 / 3}}}+\frac{1}{2} \sqrt{-\tau^{\frac{1}{3}}-\frac{9}{\tau^{1 / 3}}+\frac{16}{\sqrt{\frac{\tau^{2 / 3}+4 \tau^{1 / 3}+9}{\tau^{1 / 3}}}}+8} \cong-0.369$.

A computation of a real comprehensive triangular decomposition for $\mathcal{W}$ gives that the parameter space $\mathbf{R}$ (with coordinate $a$ ) is partitioned into two cells: $C_{0}=\{a \in$ $\mathbf{R} \mid a<\vartheta\} \sqcup\{a \in \mathbf{R} \mid a \geq 0\}$ and $C_{1}=\{a \in \mathbf{R} \mid \vartheta \leq a<0\}$. For all $a \in C_{0}$ the system $\mathcal{W}$ has no solutions and for all $a \in C_{1}$ the system $\mathcal{W}$ has exactly one real solution $\left(w_{1}, w_{2}, \lambda\right) \in \mathbf{R}^{3}$. It follows that for any valid choice of the parameter $a$ there exists a unique quadrature domain. The parameter value $a=\vartheta \in C_{1}$ corresponds to the case where $\partial \Omega$ has two cusps, as shown in Fig. 7. 


\section{Other Related Topics}

In this section, we briefly discuss some of the existing methods and constructions in the theory of quadrature domains with bearing for our above methods. The reader interested in a comprehensive picture of the area should consult one or several of the textbooks $[12,15,25,26,40,43]$.

\subsection{The Defining Polynomial of a q.d.}

Consider a quadrature domain $\Omega$ of order $n=n_{1}+\cdots+n_{m}$ corresponding to a point-functional

$$
\mu(f)=\sum_{k=1}^{m} \sum_{j=0}^{n_{k}-1} c_{k j} f^{(j)}\left(a_{k}\right) .
$$

It was shown by Aharonov and Shapiro in [2] that the boundary $\partial \Omega$ is an algebraic curve, and more precisely there exists an irreducible polynomial $P(z, w)=$ $\sum_{j, k=0}^{n} a_{j k} z^{j} w^{k}$ which is self-conjugate and normalized (i.e. $a_{j k}=\bar{a}_{k j}, a_{n n}=1$ ) such that

$$
\partial \Omega=\{z ; P(z, \bar{z})=0\} \backslash\{\text { “special points" }\}
$$

where a "special point" is an isolated solution to the equation $P(z, \bar{z})=0$. (Cf. [39])

It is convenient to refer to the polynomial $P$ in (31) as the "defining polynomial" for the quadrature domain $\Omega$. A natural problem, then, is to try to characterize the set of defining polynomials $P$ which are associated with a given point-functional $\mu$. This problem has been investigated by Gustafsson in $[19,20]$, and leads to interesting insights with respect to the uniqueness question (Q). To elucidate this, we write a defining polynomial in the form

$$
P(z, w)=\sum_{j=0}^{n} w^{j} p_{j}(z),
$$

where each $p_{j}(z)$ is a polynomial of degree at most $n$.

It is shown in [19] (cf. also [8]) that there is an explicit bijection between pointfunctionals $\mu$ (of the form (30)) and the last two polynomials $p_{n-1}, p_{n}$ which may appear in the expansion (32). As pointed out in $[8,19]$, the determination of the remaining polynomials $p_{0}, \ldots, p_{n-2}$ is generally a difficult matter. In the simply connected case, this problem is similar to the question of completely characterizing all solutions to the master formula (in Theorem 1.2), for each point-functional $\mu$.

Remark The notion of a special point is not just an artefact of the construction. For example, such points appear naturally in the process of forming smash sums of discs (i.e., each time discs "collide" as in Fig. 6 (c)) and they have the physical interpretation of stagnation points for fluid flows. We refer to $[8,20]$ for further details. 


\subsection{Connection to Laplacian Growth}

Let $t$ be a real parameter and consider a family of quadrature domains $\Omega(t)$, each obeying the quadrature identity

$$
\int_{\Omega(t)} f d A=\mu_{t}(f)=\left(c_{1}+q t\right) f\left(a_{1}\right)+c_{2} f\left(a_{2}\right)+\cdots+c_{n} f\left(a_{n}\right), \quad f \in A L^{1}(\Omega),
$$

where $q$ is a real constant, and where we take $a_{1}=0$ for simplicity. If $q>0$, we may think of $\Omega(t)$ as an expanding blob of fluid, obtained from $\Omega(0)$ by injecting fluid at the origin, at constant rate $q$. (If $q<0$, the domains contract due to suction.) The resulting evolution $t \mapsto \Omega(t)$ is known under the names "Hele-Shaw evolution" and "Laplacian growth", see e.g. $[1,26,43]$.

For values of $t$ such that $\Omega(t)$ is solid, we will write $z \mapsto \varphi(z, t)$ for the Riemann mapping $\mathbf{D} \rightarrow \Omega(t)$.

Remark Any domain $\Omega \in Q\left(\mu_{t}, A L^{1}\right)$ is by definition of finite order, and hence its boundary is part of an algebraic curve, see Subsect. 7.1. This implies that the boundary curve is smooth everywhere with the possible exception of finitely many singular points, which may be either cusps $p \in \partial \Omega$ pointing inwards (corresponding to values $p=\varphi(z), z \in \mathbf{T}$ at which $\varphi^{\prime}(z)=0$ ), or contact points $p \in \partial \Omega$ (which satisfy $p=\varphi\left(z_{1}\right)=\varphi\left(z_{2}\right)$ for two distinct points $\left.z_{1}, z_{2} \in \mathbf{T}\right)$. For solid domains, contact points are excluded.

In the following, we suppose that $\Omega(t) \in Q\left(\mu_{t}, A L^{1}\right)$ is a smoothly varying family of solid domains obeying (33) for $t$ in some suitable time-interval. A basic result relates the "time-derivative" $\dot{\varphi}(z, t):=\frac{\partial}{\partial t} \varphi(z, t)$ to the "space-derivative" $\varphi^{\prime}(z, t)=$ $\frac{\partial}{\partial z} \varphi(z, t)$.

Theorem 7.1 The conformal map $\varphi(z, t)$ onto $\Omega(t)$ satisfies Polubarinova-Galin's equation

$$
\operatorname{Re}\left[\dot{\varphi}(z, t) \overline{z \varphi^{\prime}(z, t)}\right]=\frac{q}{2}, \quad z \in \mathbf{T} .
$$

A proof can be found in the book [25, Sect. 1.4.2].

Gustafsson and Lin in [22] have studied the evolution of zeros and poles of the space-derivative $\varphi^{\prime}(z, t)$. We will now briefly indicate a different possible approach based on our basic structure theorem, Theorem 1.2. Denote by $\lambda_{i}(t)$ the points in $\mathbf{D}$ such that $\varphi\left(\lambda_{i}(t), t\right)=a_{i}$, and write $w_{i}(t)=\varphi^{\prime}\left(\lambda_{i}(t), t\right)$. Also denote $c_{1}(t)=c_{1}+q t$ and $c_{j}(t) \equiv c_{j}$ when $j \geq 2$. In view of (3), the mapping $\varphi(z, t)$ obeys

$$
\varphi(z, t)=\sum_{i=1}^{n} \frac{\overline{c_{i}(t)}}{\overline{w_{i}(t)}} \frac{z}{1-\overline{\lambda_{i}(t)} z}
$$


which gives

$$
\varphi^{\prime}(z, t)=\sum_{j=1}^{n} \frac{\overline{c_{i}(t)}}{\overline{w_{i}(t)}} \frac{1}{\left(1-\overline{\lambda_{i}(t)} z\right)^{2}}
$$

and (denoting complex conjugation by $a^{\dagger}=\bar{a}$, and abbreviating $c_{i}=c_{i}(t), \lambda_{i}=$ $\lambda_{i}(t)$, etc. $)$

$$
\dot{\varphi}(z, t)=z\left[\frac{1}{w_{1}}-\sum_{i=1}^{n}\left(\frac{c_{i} \dot{w}_{i}}{w_{i}^{2}}\right)^{\dagger} \frac{1}{1-z \bar{\lambda}_{i}}+\sum_{i=1}^{n} \frac{\bar{c}_{i}}{\bar{w}_{i}} \frac{z \bar{\lambda}_{i}}{\left(1-z \bar{\lambda}_{i}\right)^{2}}\right] .
$$

Substituting (36) and (37) in Polubarinova-Galin's equation (34), we obtain a nonlinear system of ordinary differential equations connecting the functions $\lambda_{i}(t)$ and $w_{i}(t)$ and their time-derivatives $\dot{\lambda}_{i}(t), \dot{w}_{i}(t)$. We may note from (35) that the poles of the Riemann map $\varphi(z, t)$ are given by $1 / \overline{\lambda_{i}(t)}$.

Similar sets of equations have been studied also in the case when $\varphi(z, t)=a_{1}(t) z+$ $\cdots+a_{n}(t) z^{n}$ is a polynomial in $z$, see [26, Sect. 2.1.1] and [32]. It is clear that our method applies in this case as well, but for reasons of length, we shall not pursue this issue here.

\subsection{Multi-connected Quadrature Domains}

A theory for multiply connected quadrature domains, using the Schottky double of the domain, is developed in the paper [19]. The mapping problem for such domains has been the subject of numerous investigations. In particular, for a significant class of quadrature domains (e.g. based on forming suitable smash sums of discs), the Riemann map can be constructed using the corresponding "Schottky-Klein prime function". This approach is found in the works $[10,13]$ as well as in the recent monograph [12, Ch. 11]. (The Schottky-Klein function is surveyed in the article [11].)

Other relevant works in this connection are [30] on the topology of quadrature domains, and [18], which discusses related uniqueness questions.

Acknowledgements This work was supported in part by the Anders Wall Foundation.

Funding Open access funding provided by Lund University.

Open Access This article is licensed under a Creative Commons Attribution 4.0 International License, which permits use, sharing, adaptation, distribution and reproduction in any medium or format, as long as you give appropriate credit to the original author(s) and the source, provide a link to the Creative Commons licence, and indicate if changes were made. The images or other third party material in this article are included in the article's Creative Commons licence, unless indicated otherwise in a credit line to the material. If material is not included in the article's Creative Commons licence and your intended use is not permitted by statutory regulation or exceeds the permitted use, you will need to obtain permission directly from the copyright holder. To view a copy of this licence, visit http://creativecommons.org/licenses/by/4.0/. 


\section{References}

1. Abanov, A., Mineev-Weinstein, M., Zabrodin, A.: Multi-cut solutions of Laplacian growth. Phys. D 238(17), 1787-1796 (2009)

2. Aharonov, D., Shapiro, H.S.: Domains on which analytic functions satisfy quadrature identities. J. Anal. Math. 30(1), 39-73 (1976)

3. Basu, S., Pollack, R., Roy, M.-F.: Algorithms in Real Algebraic Geometry, vol. 10. Springer, Berlin (2006)

4. Bers, L.: An approximation theorem. J. Anal. Math. 14, 1-4 (1965)

5. Chen, C.: Solving polynomial systems via triangular decomposition. PhD thesis, The University of Western Ontario (2011)

6. Chen, C., Golubitsky, O., Lemaire, F., Maza, M. M., Pan, W.: Comprehensive triangular decomposition. In: International Workshop on Computer Algebra in Scientific Computing, pp. 73-101. Springer, Berlin (2007)

7. Chen, C., Maza, M. M.: Semi-algebraic description of the equilibria of dynamical systems. In: International Workshop on Computer Algebra in Scientific Computing, pp. 101-125. Springer, Berlin (2011)

8. Crowdy, D.: Multipolar vortices and algebraic curves. R. Soc. Lond. Proc. Ser. A Math. Phys. Eng. Sci. 457(2014), 2337-2359 (2001)

9. Crowdy, D.: The construction of exact multipolar equilibria of the two-dimensional Euler equations. Phys. Fluids 14(1), 257-267 (2002)

10. Crowdy, D.: Quadrature domains and fluid dynamics. In: Peter, E., Björn, G., Dmitry, K., Mihai, P. (eds.) Quadrature Domains and Their Applications, pp. 113-129, Basel, 2005. Birkhäuser Basel (2005)

11. Crowdy, D.: The Schottky-Klein prime function on the Schottky double of planar domains. Comput. Methods Funct. Theory 10(2), 501-517 (2010)

12. Crowdy, D.: Solving Problems in Multiply Connected Domains. NSF-CBMS Regional Conference Series in Applied Mathematics: 97. SIAM, Philadelphia, USA (2020)

13. Crowdy, D., Marshall, J.: Constructing multiply connected quadrature domains. SIAM J. Appl. Math. 64(4), 1334-1359 (2004)

14. Davis, P.J.: Double integrals expressed as single integrals or interpolatory functionals. J. Approx. Theory 5, 276-307 (1972)

15. Davis, P. J.: The Schwarz function and its applications. The Mathematical Association of America, Buffalo, N. Y., 1974. The Carus Mathematical Monographs, No. 17 (1974)

16. Fejér, L., Szegö, G.: Special conformal mappings. Duke Math. J. 18(2), 535-548, 06 (1951)

17. Gardiner, S.J., Sjödin, T.: Convexity and the exterior inverse problem of potential theory. Proc. Am. Math. Soc. 136(5), 1699-1703 (2008)

18. Gardiner, S.J., Sjödin, T.: A characterization of annular domains by quadrature identities. Bull. Lond. Math. Soc. 51(3), 436-442 (2019)

19. Gustafsson, B.: Quadrature identities and the Schottky double. Acta Appl. Math. 1(3), 209-240 (1983)

20. Gustafsson, B.: Singular and special points on quadrature domains from an algebraic geometric point of view. J. Anal. Math. 51, 91-117 (1988)

21. Gustafsson, B.: On quadrature domains and an inverse problem in potential theory. J. Anal. Math. 55, 172-216 (1990)

22. Gustafsson, B., Lin, Y.-L.: On the dynamics of roots and poles for solutions of the Polubarinova-Galin equation. Ann. Acad. Sci. Fenn. Math. 38(1), 259-286 (2013)

23. Gustafsson, B., Lin, Y.-L., Roos, J.: Laplacian growth on branched riemann surfaces. To appear

24. Gustafsson, B., Shapiro, H.: What is a quadrature domain? In: Quadrature domains and their applications, volume 156 of Oper. Theory Adv. Appl., pp. 1-25. Birkhäuser, Basel (2005)

25. Gustafsson, B., Vasil'ev, A.: Conformal and potential analysis in Hele-Shaw Cell. Adv. Math. Fluid Mech. Birkhäuser Verlag (2006)

26. Gustafsson, B., Vasil'ev, A., Teodorescu, R.: Classical and stochastic Laplacian growth. Adv. Math. Fluid Mech. Springer (2014)

27. Gustafsson, B., Putinar, M.: Selected topics on quadrature domains. Phys. D Nonlinear Phenomena 235(1), 90-100 (2007)

28. Henrici, P.: Applied and computational complex analysis. Vol. 1. Wiley Classics Library. Wiley, New York, 1988. Power series-integration-conformal mapping-location of zeros, Reprint of the 1974 original, A Wiley-Interscience Publication 
29. Isakov, V.: Inverse source problems. Mathematical surveys and monographs: 34 . American Mathematical Society (1990)

30. Lee, S.-Y., Makarov, N.G.: Topology of quadrature domains. J. Am. Math. Soc. 29(2), 333-369 (2016)

31. Lemaire, F., Maza, M. Moreno, Xie, Y.: The RegularChains library. In Kotsireas, I., (ed.) Proceedings of Maple Conference, pp. 355-368. Maplesoft (2005)

32. Mineev, M.B.: A finite polynomial solution of the two-dimensional interface dynamics. Phys. D 43(23), 288-292 (1990)

33. Novikoff, P.P.: Sur le problème inverse du potentiel. Comptes Rendus (Doklady) de l'Académie des Sciences de l'URSS 18(3), 165-168 (1938)

34. Pommerenke, C.: Univalent functions. Vandenhoeck \& Ruprecht, Göttingen, 1975. With a chapter on quadratic differentials by Gerd Jensen, Studia Mathematica/Mathematische Lehrbücher, Band XXV (1975)

35. Sakai, M.: Linear combinations of harmonic measures and quadrature domains of signed measures with small supports. Proc. Edinb. Math. Soc. 42, 433-444 (1999)

36. Sakai, M.: Quadrature Domains. Springer Verlag, Berlin (1982)

37. Sakai, M.: Finiteness of the family of simply connected quadrature domains. In: Potential Theory (Prague, 1987), pp. 295-305. Plenum, New York (1988)

38. Sakai, M.: Sharp estimates of the distance from a fixed point to the frontier of a Hele-Shaw flow. Potential Anal. 8(3), 277-302 (1998)

39. Shapiro, H. S.: Unbounded quadrature domains. In: Complex Analysis, I (College Park, Md., 1985-86), volume 1275 of Lecture Notes in Math., pp. 287-331. Springer, Berlin (1987)

40. Shapiro, H. S.: The Schwarz function and its generalization to higher dimensions, volume 9 of The University of Arkansas lecture notes in the mathematical sciences. Wiley (1992)

41. Skinner, B.: Logarithmic potential theory on Riemann surfaces. ProQuest LLC, Ann Arbor, MI, 2015. Thesis (Ph.D.)-California Institute of Technology (2015)

42. Ullemar, C.: A uniqueness theorem for domains satisfying a quadrature identity for analytic functions. Technical Report TRITA-MAT-1980-37, KTH Royal Institute of Technology (1980)

43. Varčenko, A.N., Etingof, P.I.: Why the boundary of a round drop becomes a curve of order four. University lecture series: 3 . American Mathematical Society (1992)

44. Zalcman, L.: Some inverse problems of potential theory. In: Integral Geometry (Brunswick, Maine, 1984), volume 63 of Contemp. Math., pp. 337-350. Am. Math. Soc., Providence, RI (1987)

45. Zidarov, D.: Method of finding point (dipole) solutions of the potential field inverse problem. Pure Appl. Geophys. 110(1), 1918-1926 (1973)

46. Zidarov, D., Zhelev, Z.: On obtaining a family of bodies with identical exterior fields-method of bubbling. Geophys. Prospect. 18(1), 14-33 (1970)

Publisher's Note Springer Nature remains neutral with regard to jurisdictional claims in published maps and institutional affiliations. 